\title{
Message Sidedness Effects in Advertising: The Role of Yin-Yang Balancing Theory
}

\author{
Myriam Ertz ${ }^{1, *(D)}$, Myung-Soo Jo ${ }^{2}$, Fahri Karakas ${ }^{3}$ and Emine Sarigöllü $^{2}$ (D) \\ 1 LaboNFC, Department of Economics and Administrative Sciences, University of Quebec at Chicoutimi, \\ Saguenay, QC G7H 2B1, Canada \\ 2 Desautels Faculty of Management, McGill University, Montreal, QC H3A 1G5, Canada; \\ myung-soo.jo@mcgill.ca (M.-S.J.); emine.sarigollu@mcgill.ca (E.S.) \\ 3 Norwich Business School, University of East Anglia, Norwich NR4 7TJ, UK; f.karakas@uea.ac.uk \\ * Correspondence: Myriam_Ertz@uqac.ca; Tel.: +1-418545-5011
}

Citation: Ertz, Myriam, Myung-Soo Jo, Fahri Karakas, and Emine Sarigöllü. 2021. Message Sidedness Effects in Advertising: The Role of Yin-Yang Balancing Theory. Social Sciences 10: 229. https://doi.org/ 10.3390/socsci10060229

Academic Editor: Pierre Desrochers

Received: 23 April 2021

Accepted: 10 June 2021

Published: 15 June 2021

Publisher's Note: MDPI stays neutral with regard to jurisdictional claims in published maps and institutional affiliations.

Copyright: (c) 2021 by the authors. Licensee MDPI, Basel, Switzerland. This article is an open access article distributed under the terms and conditions of the Creative Commons Attribution (CC BY) license (https:// creativecommons.org/licenses/by/ $4.0 /)$.

\begin{abstract}
Past research has shown mixed results regarding the persuasiveness of two-sided messages. Various underlying constructs were suggested to explain the differences in results. This study draws on the Yin-Yang Balancing (YYB) theory and the construct of tolerance for contradiction (i.e., the tolerance for inconsistency and resolution among contrasts) to explain differences in the effectiveness of two-sided ads. The study consisted of a cross-cultural survey involving Easterners, who hold typically higher tolerance for contradiction, with Westerners characterized by a lower tolerance for contradiction. A series of analyses of variance (ANOVAs) were conducted to explore the difference between both groups on key variables. Structural equation modeling (SEM) tested the proposed conceptual model as a whole and for both groups, highlighting key cross-cultural differences. Additionally, the PROCESS macro was used to test the mediation effects posited in the model. The findings showed that although the tolerance for contradiction does not directly impact purchase intentions, it exerts both direct and indirect effects on purchase intentions through credibility and attitudes for Easterners but not for Westerners. The findings offer important theoretical and managerial implications: Two-sided ads are more effective to consumers with a higher tolerance for contradiction (e.g., Easterners) versus consumers with a lower tolerance for contradiction (e.g., Westerners).
\end{abstract}

Keywords: two-sided messages; Yin-Yang balancing theory; tolerance for contradiction; cross-cultural study; purchase intentions; attitudes; credibility; consumers; advertisement; mediation analysis

\section{Introduction}

Two-sided messages are communications in which a source integrates both positive and negative sides about a product while favoring one side to persuade the audience (Hovland 1954). In contrast, one-sided messages only provide positive information (Crowley and Hoyer 1994). Whether two-sided messages are effective, the debate is still unresolved and ongoing (Eisend 2013; Küster and Eisend 2016; Pizzutti et al. 2016; Huertas and Hanna 2020; Cornelis et al. 2020; Kim 2020). Although various explanations have been offered for the discrepancy in empirical findings, such as methodological differences (e.g., Crowley and Hoyer 1994; Eisend 2006) or absence of a specific theory within the context of marketing information processing (e.g., Crowley and Hoyer 1994), past research has not studied the possibility that such differences may stem from core cultural characteristics.

Past research on the effectiveness of two-sided messages mainly originated from Western countries and showed inconsistent results (Kamins and Marks 1987; Trifts and Häubl 2003; Eisend 2007, 2008, 2010; Cornelis et al. 2012, 2015; Pizzutti et al. 2016). A considerable stream of research, including Allen's (1991) meta-analysis, demonstrates the enhanced persuasiveness of two-sided messages compared to their one-sided counterparts (Swinyard 1981; Etgar and Goodwin 1982; Lang et al. 1999; Eisend 2007, 2008, 2010; Cornelis et al. 2015; Pizzutti et al. 2016), which often results in superior purchase intentions 
(Eisend 2007). In sharp contrast, other studies have failed to reach similar conclusions (Belch 1983; Hunt and Smith 1987; Park and Hastak 1994) or provided a multiplicity of moderating variables which nuanced two-sided message effectiveness such as the need for cognition (NFC) (Qi et al. 2010a, 2010b; Kao 2011) and time pressure (Kao 2011). In a review of Allen's meta-analysis, O'Keefe (1999) warns that conclusions based on this study should not be accepted uncritically. He argues that the misclassification of studies within the meta-analysis and its inadequate literature search provides sufficient reason to question Allen's conclusions. Moreover, it appears that a two-sided message actually decreases consumers' purchase likelihood, especially when an important attribute is being disclaimed (Pizzutti et al. 2016). In light of this diversity of results, Eisend (2006) and Cornelis et al. (2015) suggested further investigations into the influence of moderators, mainly since moderators of negative information remained unknown (Hair and Bond 2018).

Recent studies have started to investigate such moderators as self-confidence and demonstrate their effect on the link between two-sided messages and purchase intentions (e.g., Huertas and Hanna 2020). Other intrapsychic variables used as moderators include the need for cognition (Qi et al. 2010a, 2010b; Kao 2011). Another stream of studies used message characteristics as moderators. Cornelis et al. (2014) showed how refutation counters the adverse effects of ambivalence in two-sided messages. On the other hand, Campos (2018) found that humorous warmth is the principal factor enhancing message persuasion, source credibility, and attitude. We propose that such intrapsychic and ad characteristics are not sufficient to explain the effectiveness of two-sided messages. Instead, we converge with an emerging stream of research that signifies the importance of contextual factors, such as culture, in assessing the effectiveness of message sidedness in advertising (Qi et al. 2010a, 2010b).

Consequently, we propose that culture is an essential factor for understanding the effectiveness of two-sided messages. Its inclusion enables us to comprehend the mechanism underlying two-sided message persuasiveness. More specifically, the East Asian philosophical framework of Yin-Yang Balancing (YYB) theory illuminates a critical underlying mechanism that explains why two-sided advertisements may work or not: the tolerance for contradiction, that is, the tolerance for inconsistency and resolution among contrasts (Li 2012, 2016; Fang 2012). Yin Yang is an indigenous Chinese philosophy that embodies paradox and harmony through the juxtaposition of two opposite and dynamic cosmic energies: Yin (representing 'female' energy, water, earth, the moon, the night, darkness) and Yang (denoting 'male' energy, fire, the sky, the sun, the day, brightness) (Pauluzzo et al. 2018; Pauluzzo 2020). The small dots within each of the energies (black in white and white in black) symbolize how these two forces are co-existing, complementary, and interconnected for a harmonious cognition (Chin et al. 2018). Yin Yang philosophy has influenced the Chinese way of life (including medicine, arts, literature, politics, culture, business, and consumer behavior) for centuries (Fang and Faure 2011; Li et al. 2018).

This influence is often reflected in the form of dualist and paradoxical thinking (Fang 2012; Li 2016), such as the concept of weìi $\bar{\imath}$ (the Chinese word meaning 'a point where things happen or change', which implies both 'danger' and 'change point' and a balance of both). Seen from a Yin Yang perspective, two-sided messages provide both favorable and opposing arguments for a product or service. Eastern customers, in turn, may feel more comfortable with paradoxical and potentially incompatible advertising messages because those messages befit well their cultural predispositions towards balancing, which is rooted in Yin-Yang tradition (Fang and Faure 2011). Past research showed preliminary evidence that two-sided messages increase the helpfulness of online reviews, among EastAsian customers, especially for expert consumers (e.g., Chen 2016). Research suggests that two-sided (one-sided) reviews are more (equally) helpful than (to) one-sided (two-sided) ones for search goods if the reviewer is an expert (novice). For experience goods, one-sided are more (equally) helpful than (to) two-sided reviews if the reviewer is an expert (novice) (Chen 2016). These results were confirmed by Li et al. (2020). They demonstrated that review sidedness is positively related to perceived helpfulness, while that effect is stronger 
for search products than for experience products. Huertas and Hanna (2020) also found a similar effect of message sidedness on purchase intention while further demonstrating that high (low) self-confidence individuals are more likely to increase (decrease) the purchase when exposed to a two-sided message instead of a one-sided message.

Thus, we seek to answer the following research question: how effective two-sided advertising messages are for Chinese and American consumers in light of the Yin-Yang Balancing Theory? To answer this question, this study proposes the 'tolerance for contradiction' (i.e., need for balancing) as a significant explanatory factor for the effectiveness of two-sided ads and endeavors to make several contributions to the literature.

The overarching contribution resides in an improved understanding of international consumer behavior by proposing a Yin Yang perspective to inquire about the effectiveness of two-sided advertising messages in cross-cultural settings. More specifically, we answer the previous call in the literature (e.g., Qi et al. 2010a, 2010b) to assess contextual factors, such as culture, in the study of two-sided messages. Second, although past research has used the NFC-Elaboration Likelihood Model (ELM) to explain cultural differences in twosided message persuasiveness (Qi et al. 2010a, 2010b), the findings have been inconsistent (Kao 2011). Instead, we draw on the Yin-Yang Balancing Theory and the tolerance for contradiction construct, which is deeply rooted in core cultural differences, to explain why different reactions to two-sided messages might occur. Third, we explore how and when two-sided messages are effective and consequently aid in managers' decision-making process, especially those engaged in advertising across different cultural contexts.

The remainder of the paper is organized as follows: the conceptual framework presents the Yin-Yang Balancing theory before developing hypotheses. We then present our methodology and results. The paper concludes by delineating theoretical and managerial implications and limitations, and avenues for future research.

\section{Conceptual Framework}

\subsection{The Yin-Yang Balancing Theory as an Explanation for Cultural Differences}

We define the tolerance for contradiction, or preference for balance, as the tolerance for inconsistency and resolution among contrasts (Li 2012; Fang 2012). This paper examines the tolerance for contradiction as an essential measure of cultural difference. The tolerance for contradiction differs significantly from culture.

Although Chinese culture and logic might differ from those of other Asian countries or regions, it appears that Easterners, in general, and the Chinese in particular, have a higher tolerance for contradiction (Fang 2012; Li 2012, 2016). This higher tolerance for contradiction expressed by Easterners can more generally be explained by the Yin-Yang Balancing (YYB) theory. This theory offers an alternative method to interpret cultural differences/similarities to those based on Hofstede's interpretation of culture (Fang 2005-2006; Li 1998, 2016) because this interpretation of culture is characterized by different flaws (McSweeney 2015). Beyond the oversimplistic Hofstedian characterization of the collectivist vs. individualistic divide between Easterners and Westerners, Chinese culture, in particular, is holistic, duality-based, and dynamic (Peng and Nisbett 1999), which can be explained by the Yin-Yang Balancing (YYB) theory (Li 2012; Fang 2012). The concept of Yin-Yang refers to an ancient Chinese philosophical principle, which holds that there are no absolute separations between opposites (Cooper 1990; Mattsson and Tidström 2015; Pauluzzo 2020). The Yin-Yang principle intrinsically refers to paradox, duality, unity in diversity, harmony, and change (Chen 2002).

Yin-Yang thinking bears some resemblance to the dialectical thinking in the West (Peng and Nisbett 1999). However, the YYB is an open system "to accommodate a balance between 'either/or' and 'both/and.' It is highly distinctive from Aristotle's formal logic, which is mechanistic and reductionist due to its absolute denial of potential contradictions with a permanent 'either/or' (but never 'both/and'). It is also different from Hegel's dialectical logic, which is ultimately mechanistic and reductionist due to its absolute need for the resolution of transitory contradictions with a temporary 'both/and' but ultimate 
'either/or,' so [we] term it 'both/or'"' (Zolfani et al. 2013, p. 157). This implies that the Chinese YYB framework, which infuses Eastern thinking, accommodates strong forms of contradiction (Nisbett 2003; Li 2012; Pauluzzo 2020). In contrast, Aristotle's formal logic and Hegel's dialectic logic, as examples of Western thinking, are dualist and do not accommodate contradictions.

The Yin-Yang balancing follows a holistic, dynamic, and duality structure (Li 2016), according to which the conflicting yet complementary elements/values co-exist and mutually transform into each other in an organic and unified whole (Li 1998; Tian 2002). This dialectical relationship with partial trade-offs and synergies between opposite elements/values is strictly related to the concept of a paradox, characterized by "the simultaneous presence of contradictory, even mutually exclusive elements" (Cameron and Quinn 1988, p. 2). In this regard, the YYB fully embraces paradox, recognizing that individuals are both Yin and Yang (Fang 2005-2006). In this context, the YYB, by considering opposites as partially conflicting and partially complementary (Jing and Van de Ven 2014), implicitly adopts an 'either/and' approach, which contrasts both the 'both/and' logic that treats opposites as entirely complementary without conflict, and the 'either/or' system that views opposites as fully conflicting without complementarity ( $\mathrm{Li}$ 2016) As a matter of fact, the YYB frame of thinking has tremendous implications and applications for exploring complex phenomena (Zolfani et al. 2013) and potentially for advertising. Table 1 explains the core arguments of the YYB theory and outlines its implications for advertising messages.

Table 1. Applying Yin-Yang perspective for two-sided advertising.

\section{Theme}

Enhancing credibility through acknowledging opposite arguments as a vital and dynamic element

Maintaining trust

\section{Philosophy and Corresponding Messages/Strategies}

There are two sides to every coin. A phenomenon cannot be complete unless it has two opposite elements. Yin-Yang is a holistic and dialectical worldview that thrives on the vital duality of opposing elements. Opposite elements are seen as dynamic as they mutually transform into each other in a balancing process. Advertising messages that include opposites might still be seen as holistic and powerful; therefore, viewed as credible in Eastern cultures.

In Yin-Yang perspective, the paradox is seen as a necessary element of a rich and meaningful life. A paradox does not comprise independent and exclusive opposites but consists of interdependent and complementary opposites. Advertising messages acknowledging paradox and dynamism are seen as reflective of the messy realities of life.

Taoist Yin-Yang thinking emphasizes focusing attention on both sides of any claim or any situation. The inclusion of negative information in advertising messages might lead to better trust. Since consumers perceive claims to be associated with actual characteristics of the product rather than the advertiser's desire to sell the product, they might better trust two-sided advertising messages.

Yin and Yang are never static, but they are in a constant interchanging balance. Each force carries the seeds of the other one (i.e., the counterforce). The seeds of the Yin lie within the Yang and vice versa. Giving up or constraining one of these forces will

Achieving harmony through balancing disrupt the whole balance. Besides, the "seeds" are the sources of tension for trade-off as well as complementarity for synergy, underlying the dynamic process of interaction and inter-transformation between the opposite elements. Thus, harmony in life can be achieved through constant adaptation and balancing. Advertising messages acknowledging and balancing these two sides will attract more attention and trust.

Two-sided advertising messages might better capture the attention of consumers due to their novelty. The inclusion of both positive and negative messages might better differentiate the advertisement in the midst of abundant claims and exaggerations. Nuances might be perceived as more original and might evoke more favourable attitudes from consumers. 
Table 1. Cont.

\begin{tabular}{cc}
\hline Theme & Philosophy and Corresponding Messages/Strategies \\
\hline $\begin{array}{c}\text { Incorporating humour and rhythm of } \\
\text { life }\end{array}$ & $\begin{array}{c}\text { Tao mirrors life as a rhythmic and nonlinear process. Life is ironic, and it is full of } \\
\text { contradictions and tensions. A dose of light-hearted humour might be useful as a } \\
\text { coping mechanism. Humour offers the chance to confront tensions in a less threatening } \\
\text { and more playful fashion. Advertising messages that are playful and satirical might } \\
\text { better capture the rhythm of life through normalizing the situation, and they might also } \\
\text { help ease the tensions among necessary contradictions. }\end{array}$ \\
\hline $\begin{array}{c}\text { Achieving immunity against counter } \\
\text { claims and competitors }\end{array}$ & $\begin{array}{c}\text { The inclusion of negative information in advertising messages might function like a } \\
\text { vaccination against competitors' doubt and counterarguments. Countering and refuting } \\
\text { negativity early on might inoculate consumers and strengthen the core message. }\end{array}$ \\
\hline
\end{tabular}

Source: Chen (2008); Fang (2012, 2014); Fang and Faure (2011); Li (2012); Peng and Nisbett (1999).

To better show how a two-sided advertising is framed by integrating YYB theory, Figures 1 and 2 show the Chinese philosophy of balance, and a two-sided sample advertising from China, respectively. Figure 1 conveys the foundations of the dynamic duality-based principle that is core to YYB. Specifically, it reconciles the two opposites of the metaphysical (i.e., the heart and lofty thoughts) and the physical (i.e., feet on the ground) into a harmonious whole. In other words, instead of clashing the two opposites due to their differences, it reconciles them as complementary such that wholeness could not be achieved with either one of them missing. Figure 2 embodies the commercial exploitation of that balancing principle in advertising. The advertising suggests that beauty is not the result of product use alone (as many commercials tend to puffer), but instead it is the outcome of subtle combination of both "natural beauty" (i.e., traits, body features) and "artificial beauty" (i.e., the cosmetic) which combine holistically and synergistically to magnify beauty even more.

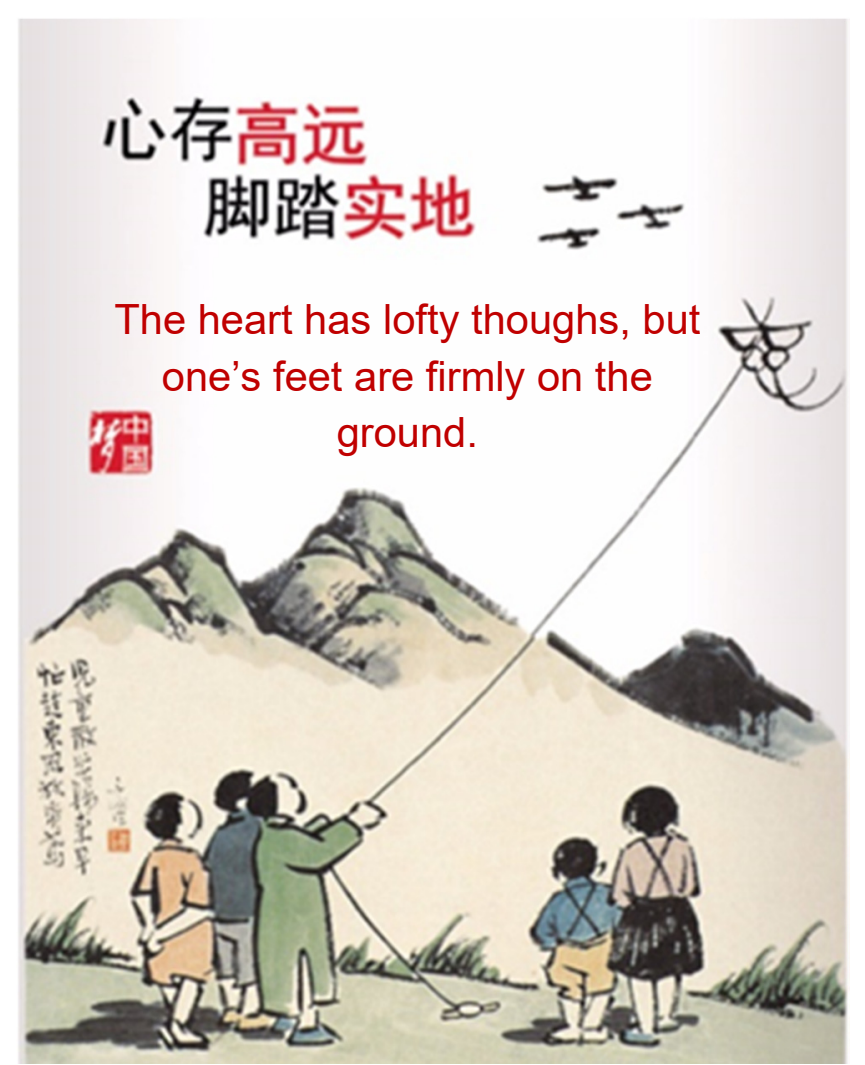

Figure 1. Sample painting reflecting the Chinese philosophy of balancing. Source: https://www. thechinastory.org/wpcontent/uploads/2013/10/F1_1c_feng_zikai_kite.jpg (accessed on 11 June 2021). 


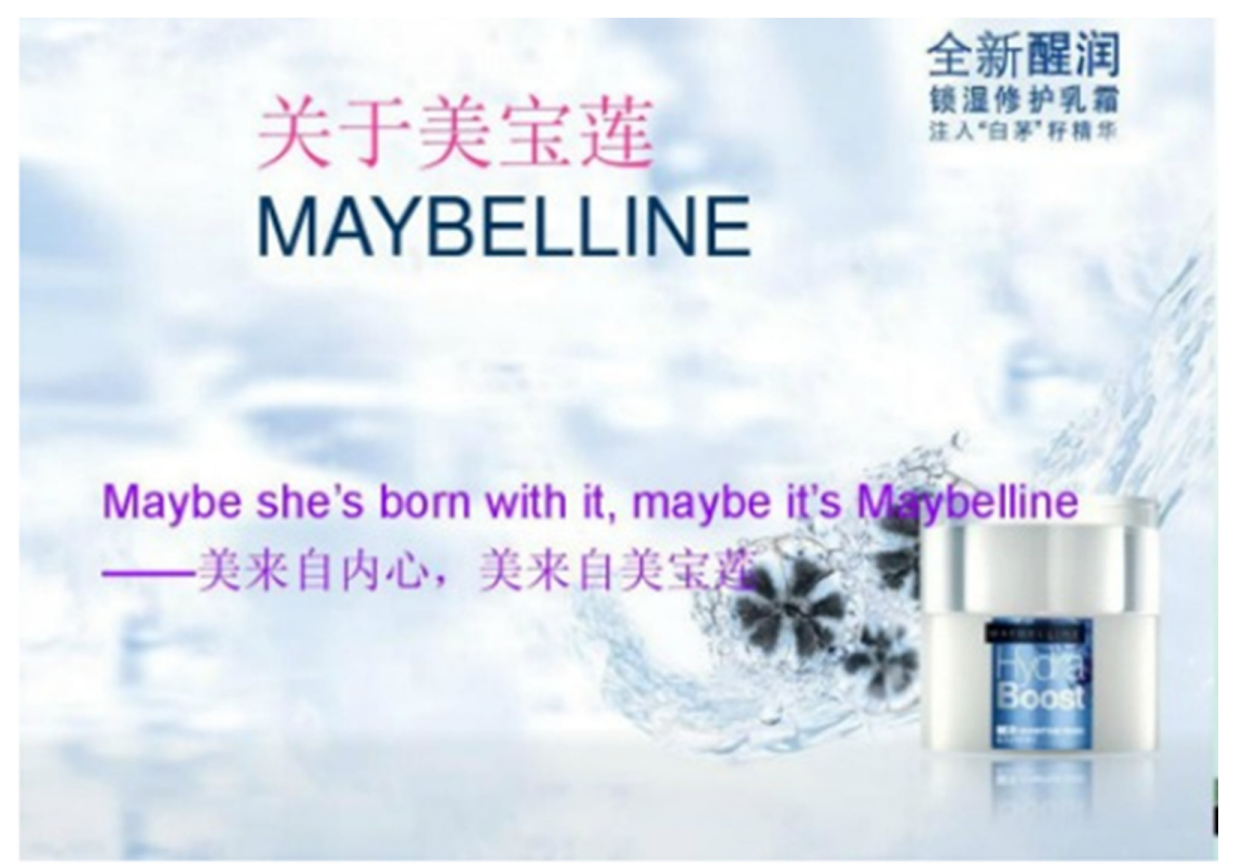

Figure 2. Sample two-sided advertising from China. Source: http:/ /www.whatsonweibo.com/ chineseadslogans/ (accessed on 11 June 2021).

Consequently, in contrast to Westerners, Easterners may accommodate relatively well counter-intuitive messages such as two-sided messages. In fact, because of their higher tolerance for contradiction, Easterners may tend to avoid extremes that are typically salient in advertising; they refrain from making black or white evaluations of objects, such as products in an advertisement. They can also be described as wanting to maintain harmony in all aspects of their lives. Yet, one-sided advertising elicits the positive about an object while deliberately disregarding the negative, thus not providing a comprehensive evaluation of the object. This approach is close to puffery (Kamins and Marks 1987). One-sided advertising may trigger higher counter-argumentation and derogation due to the restrictive perspective that it entails (Kamins and Assael 1987). Overall, two-sided messages may be more effective for Easterners who have a higher tolerance for ambiguity due to their higher tolerance for contradiction. In contrast, this effect will be less significant for Westerners.

\subsection{Hypothesis Development}

This paper examines the effectiveness of two-sided advertising messages crossculturally. We propose that Easterners' and Westerners' differentiated worldview due to their respective frames of thinking and philosophical discourses (Fang 2012; Li 2012) might impact their response to two-sided messages.

Therefore, this study uses the YYB theoretical framework and the construct of tolerance, encapsulated in tolerance for contradiction as a key constitutive factor of that theory (Chen 2002, 2008; Li 1998, 2008), as the theoretical framework of this study. This theory has already been adopted and adapted in the context of academic research (e.g., Zolfani et al. 2013) - albeit in other domains-and therefore, it is highly relevant and justified to use that framework in the current study. More specifically, while we posit a difference in tolerance for the contradiction between Chinese and Americans, we further posit that those differentiated levels of tolerance for contradiction reverberate throughout other key constructs that have been shown to predict purchase intentions, such as credibility and attitude. The following paragraphs explain in more detail the theoretical foundations underlying the study.

First, we suggest that, under the influence of the duality-based thinking induced by the YYB cognitive frame (Peng 1997), Eastern consumers tend to avoid extremes; they refrain from making black or white evaluations of people, objects, and events (Peng and 
Nisbett 1999). Instead, they have a higher level of acknowledgment and acceptance for paradox, change, duality, unity in diversity, and harmony, offering a more holistic approach to problem solving by acknowledging multiple views and perspectives (Chen 2002). This duality-based thinking is a balancing process consisting of mutually embracing opposite elements because they are considered complementary, compatible, and relative, instead of separate, incompatible, and absolute (Fang 2012; Li 2012; Zolfani et al. 2013). The notion of duality and balance derives from YYB theory, a unique cognitive frame in East Asia that originated in China (Chen 2002, 2008; Li 1998, 2008) and has thus influenced Chinese thinking style and culture. It is different from Aristotle's formal logic or Hegel's dialectal logic, which rejects the coexistence of contradiction ( $\mathrm{Li}$ 2012). While YYB prevails in East Asia, formal logic is tantamount to the Western thinking style and should decrease Westerners' tendency to balance contradiction, duality, and paradox (Zolfani et al. 2013). We expect the following:

Hypothesis 1. Eastern consumers have a higher tolerance for contradiction than Western consumers.

Past research proposed that the persuasiveness of two-sided messages is essentially driven by increased source credibility (Kamins et al. 1989; Smith and Hunt 1978; Swinyard 1981; Uribe et al. 2016). Moreover, two-sided ads are typically paradoxical because they involve "contradictory yet interrelated elements-elements that seem logical in isolation but absurd and irrational when appearing simultaneously" (Lewis 2000, p. 760). It may appear logical to see promotion and puffery in an ad (e.g., Kamins and Marks 1987). In contrast, criticism is more likely to be found in a consumer association report or a scientific journal article. Hence, the paradox. Yet, calling this a paradox is a question of perspective. From a linear logic paradigm, as characterizes the Western world, paradoxes carry negative connotations and will be disliked by the Western mind (Fang 2012). Even though the Western world may also have developed a form of dialectal logic (e.g., Hegel, Marx), resulting in such concepts as coopetition, or glocalization (Li 2012), the "Western dialectal logic fails to truly transcend the 'either/or' thinking because it still regards paradox as a problem to be solved" (Li 2008, p. 416). However, the source of a two-sided ad might become more believable (Etgar and Goodwin 1982. The level of enhanced source believability will remain lower than Easterners' due to Westerners' lower tolerance for contradiction. This lower tolerance for contradiction will not compensate for the higher perception of confusion associated with the ad source. The source may be perceived as manipulative, lacking clarity, and ultimately credibility. Therefore, the lower level of tolerance for contradiction for Westerners' balance will negatively impact their perception of source credibility.

Conversely, the YYB induces paradoxical thinking as a fundamental philosophical principle (Peng and Nisbett 1999). Henceforth, people who are influenced by the YYB frame of thought, i.e., Eastern consumers, might find an advertisement characterized by mutual interdependence and contradictions (e.g., two sides of the same coin) more believable and credible because it is in tune with one's worldview and frame of thinking (Zolfani et al. 2013). We, therefore, suggest that:

Hypothesis 2a. Tolerance for contradiction is positively related to the perceived credibility of the advertisement.

Hypothesis $\mathbf{2} \mathbf{b}$. The positive relationship between the tolerance for contradiction and credibility is stronger for Eastern consumers than Western consumers.

In line with past research (Spears and Singh 2004), we distinguish between attitude towards the advertising vs. the brand and consider both mediators of the tolerance for contradiction-purchase intention relationship. Past studies demonstrated that attitude to-wards the advertising is an antecedent to attitude towards the brand (Eisend 2007). Furthermore, the tolerance for contradiction contributes to positive attitudes towards 
advertising through enhanced credibility (Kamins et al. 1989). In line with our previous developments about YYB, the YYB framework as a Chinese duality thinking frame induces a higher tolerance for contradiction. Increased tolerance for contradiction leads to considering paradoxes as interdependent opposites rather than exclusive opposites (Chen 2002). Therefore, advertisements and brands bearing similarities with duality thinking by combining opposites, as two-sided advertisement, will foster favorable attitudes among individuals with higher needs for balance. With YYB being a unique Chinese reasoning style, this effect will be stronger for Easterners than for Westerners. Thus:

Hypotheses 3a. The tolerance for contradiction is positively related to attitude towards advertising.

Hypotheses $3 \mathbf{b}$. The tolerance for contradiction is positively related to attitude towards the brand.

Hypothesis 3c. The positive relationship between tolerance for contradiction and attitude toward the advertising and the brand is stronger for Eastern than for Western consumers.

We assess message effectiveness by purchase intentions since purchase is the ultimate goal of a marketer through advertising. Extant literature further emphasizes the link between attitudes towards the ad and purchase intentions and attitudes towards the brand and purchase intentions (Moon and Sprott 2016). As mentioned above, the persuasiveness of two-sided messages is essentially driven by an increase in source credibility. Enhanced credibility contributes to more positive attitudes towards the advertising and the brand (Chebat et al. 2001). Furthermore, an essential outcome of enhanced credibility is that it creates higher purchase intentions (Uribe et al. 2016). Collectively, these past studies suggest that enhanced credibility will positively affect both attitudes towards the advertising and the brand being advertised and purchase intentions.

In line with the YYB framework, this effect will be much stronger for Easterners than for Westerners. As duality-based thinkers with a higher tolerance for ambivalence and ambiguity (Peng and Nisbett 1999), Easterners perceive a source using paradoxical statements as more credible. This enhanced credibility of the source will further strengthen the empirically verified impact of source credibility on attitudes (Chebat et al. 2001) and purchase intentions (Uribe et al. 2016). We thus hypothesize:

Hypotheses 4a. Credibility is positively related to the advertising.

Hypotheses $4 \mathbf{b}$. Credibility is positively related to attitude towards the brand.

Hypothesis 4c. The positive relationship between credibility and attitude towards the advertising and the brand is more substantial for Eastern consumers than for Western consumers.

Hypothesis 5a. Credibility is positively related to purchase intention.

Hypothesis $\mathbf{5 b}$. The relationship between credibility and purchase intention is stronger for Eastern consumers than for Western consumers.

Attitude toward the ad is a mediator of consumer choice (Shimp 1981), but more significantly, it predisposes attitude toward the brand (Gardner 1985; Mittal 1990). Therefore, we posit the following:

Hypothesis 6a. Attitude toward the ad is positively related to attitude toward the brand.

Hypothesis $\mathbf{6 b}$. The positive relationship between attitude towards the advertising and attitude toward the brand is stronger for Eastern consumers than Western consumers.

Furthermore, in line with the theory of reasoned action (Hale et al. 2002) and the theory of planned behavior (Ajzen 1991), attitude is a strong predictor of behavioral intentions, and therefore: 
Hypothesis 7a. Attitude toward the ad is positively related to intentions.

Hypothesis $7 \mathbf{b}$. The positive relationship between attitude toward the ad and intentions is stronger for Eastern consumers than Western consumers.

Hypothesis 8a. Attitude toward the brand is positively related to intentions.

Hypothesis $\mathbf{8 b}$. The positive relationship between attitude toward the brand and intentions is stronger for Eastern consumers than Western consumers.

Finally, we propose that balance is related to purchase intentions through a mediational model involving credibility and attitude towards the ad and the brand as mediators. While we explained how the tolerance for contradiction is related to source credibility and attitudes, both attitudes (Ajzen 1991) and source credibility (Chebat et al. 2001) are related to purchase intentions. Given the empirical support for significant relationships between the constructs mentioned above in the abovementioned hierarchy of effects, we posit that the tolerance for contradiction may exert an indirect (i.e., mediation) impact on purchase intentions through an intrapsychic process. In line with YYB theory, the relationships between the antecedents will be stronger because of the initially higher tolerance for contradiction (Chen 2002; Li 2012; Fang 2012). The stronger acceptance of unequivocal messages, such as two-sided advertising, typically yielded by YYB duality-based thinking (Peng and Nisbett 1999), will ultimately reverberate on subsequent mediational constructs affecting more positively purchase intentions. Therefore, the process differs between Eastern and Western consumers since we expect stronger mediational effects of tolerance for contradiction on intentions to buy for Eastern consumers than Western ones.

Hypothesis 9a. The relationship between the tolerance for contradiction and purchase intentions is fully mediated by credibility, attitude towards the advertising, and attitude towards the brand.

Hypothesis $\mathbf{9 b}$. The indirect effect of the tolerance for contradiction on purchase intentions is stronger for Eastern consumers than for Western consumers.

The conceptual model is shown in Figure 3.

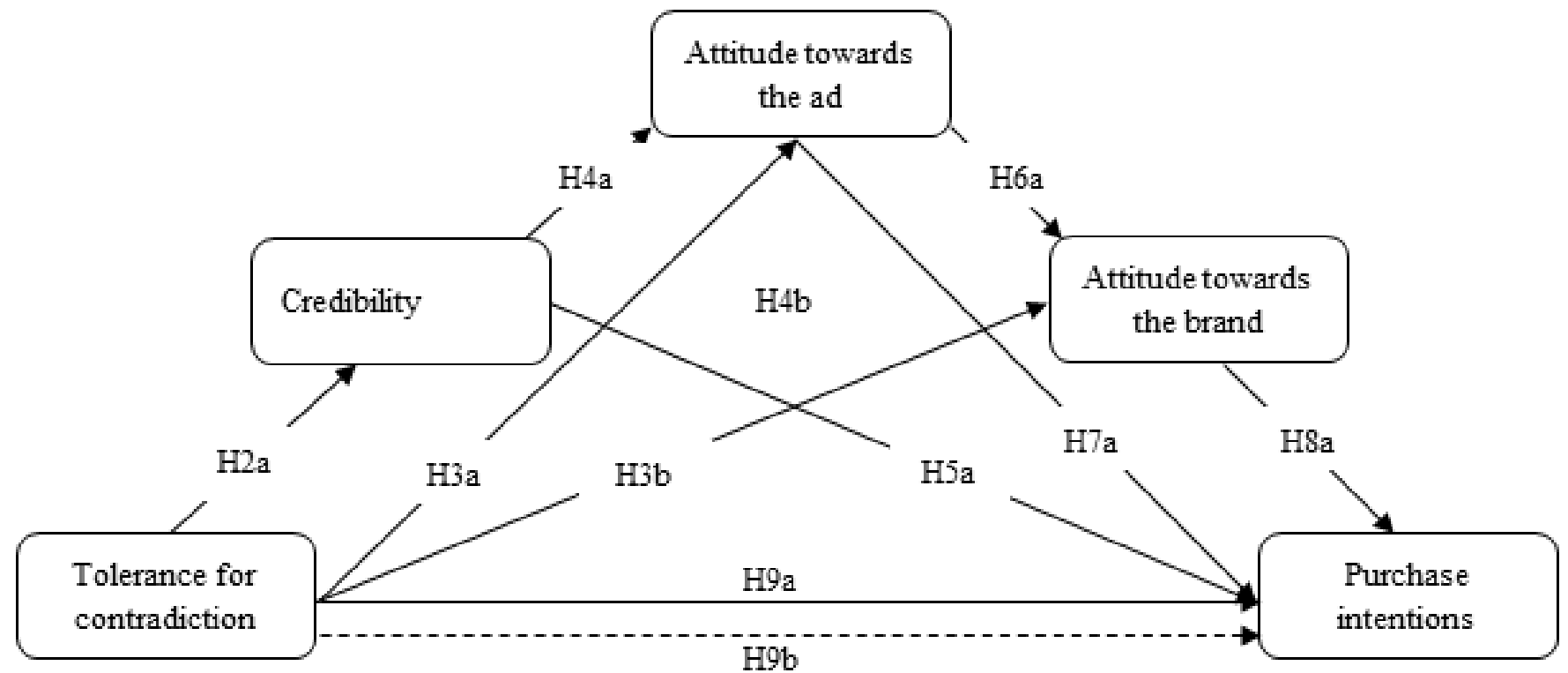

Figure 3. Conceptual model. 


\section{Method}

\subsection{Study Procedure}

The study was conducted in China as an Eastern country and the US as a Western country. Several pretests for the questionnaire were implemented. Five bilingual assistants fluent in Chinese and English were hired to generate several advertisements suitable for both China and the US. Upon testing the advertising copies on 15 Chinese and US participants, respectively, one copy was identified as the most liked and suitable for both countries-“ABC Cough Medicine" with the following two-sided message: "Tastes terrible. But scientifically proven as No.1 cough medicine." The advertising copies in both English and Chinese are provided in Figure 4. Both countries' participants showed similar liking of the advertisement copy as there were no significant differences in their ratings on the seven-point scale liking measures. We pretested the questionnaire with 30 participants from China and 21 from the US using the selected copy.

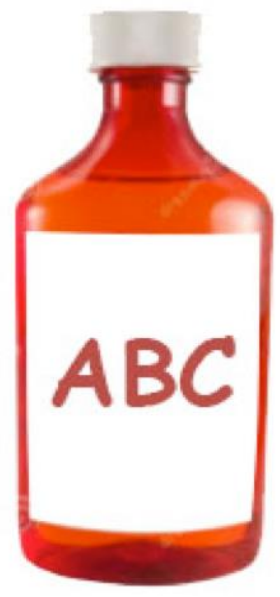

味道糟透了。 但经科学证明其为 止咳第一药。

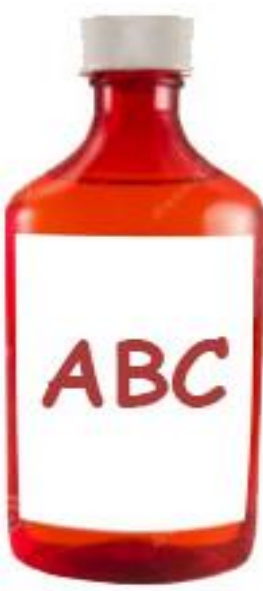

Tastes Terrible. But scientifically proven as the \#1 Cough Medicine.

Figure 4. Advertising copies.

Consequently, ambiguous wording was corrected, and the survey was revised and shortened. The pretest also confirmed that both American and Chinese groups well accept the same anonymous brand name ABC. After revision, the survey was implemented online by Sojump in China and MTurk in the US.

Despite their nonprobabilistic sampling nature, the data from these crowdsourcing sources are as reliable as those obtained via traditional survey methods (e.g., Kees et al. 2017). The survey was submitted online, and eligible respondents (18 years old and over) from the Sojump platform in China and the MTurk platform in the US were invited to participate in the survey. More specifically, potential survey respondents of both platforms were presented with a short description of study objectives, compensation amount, duration, and a link to click on. This presentation was prompted on their daily task feed from which they can choose. Since survey participants are self-selected, the researchers have no information on the total number of participants who were invited compared to the total number of participants who filled in the survey. Only the total number of completed surveys (determined by the researcher) is available to researchers, making it impossible to compute the response rate. In total, 206 respondents from the USA and 268 respondents from China provided data for 474 responses. After removing incomplete or invalid questionnaires, the final sample consisted of 423 respondents from the USA $(n=196)$ and China $(n=227)$. Table 2 shows the demographic characteristics of both samples. 
Table 2. Demographic characteristics.

\begin{tabular}{ccc}
\hline Characteristics & USA $(\boldsymbol{n}=\mathbf{1 9 6})$ & China $(\boldsymbol{n}=\mathbf{2 2 7})$ \\
\hline Sex & & \\
Male & $58.5 \%$ & $40.3 \%$ \\
Female & $41.5 \%$ & $59.7 \%$ \\
\hline Age & & \\
Under 25 & $17.0 \%$ & $24.3 \%$ \\
$26-35$ & $47.2 \%$ & $44.7 \%$ \\
36-45 & $19.7 \%$ & $17.0 \%$ \\
$46-55$ & $8.7 \%$ & $14.1 \%$ \\
Over 56 & $7.4 \%$ & $0.0 \%$ \\
\hline Education & & \\
High school graduate & $45.0 \%$ & $2.9 \%$ \\
Bachelor's degree & $45.9 \%$ & $52.4 \%$ \\
Postgraduate degree & $9.2 \%$ & $36.9 \%$ \\
\hline Ethnicity & & \\
North American & $65.9 \%$ & $0.0 \%$ \\
European & $19.2 \%$ & $0.0 \%$ \\
African & $1.7 \%$ & $0.0 \%$ \\
Latino & $4.4 \%$ & $0.0 \%$ \\
East Asian & $7.0 \%$ & $100.0 \%$ \\
South Asian & $1.3 \%$ & $0.0 \%$ \\
Middle Eastern & $0.4 \%$ & $0.0 \%$ \\
\hline Prevalent culture & & \\
Western & $92.4 \%$ & $0.0 \%$ \\
Eastern & $7.6 \%$ & $100.0 \%$ \\
\hline
\end{tabular}

\subsection{Measures}

Respondents first looked at a print advertisement for a hypothetical cough medicine then completed an online questionnaire that consisted of items measuring key constructs. All items were measured on a seven-point Likert scale (from 1 "strongly disagree" to 7 "strongly agree"), unless stated otherwise below. The dependent variable, purchase intentions, were measured with three items $(\alpha=0.923$; Madden et al. 1988; Spears and Singh 2004). The independent variable, tolerance for contradiction, was measured with four items ( $\alpha=0.817$; Graham 1986; Li 1998, 2012; Peng and Nisbett 1999). There were three mediators. First, the advertiser's credibility was measured by three items $(\alpha=0.922$; Beltramini 1988; Greer 2003; MacKenzie and Lutz 1989). Second, attitude towards the advertising, and third, attitude towards the brand were each assessed with three items (bad/good, negative/positive, unappealing/appealing), with the question: "please describe your overall feelings toward the advertisement you just saw" ( $\alpha=0.895$ and $\alpha=0.799$; Madden et al. 1988; Spears and Singh 2004). All items used to measure the dependent, independent, and mediator variables are displayed in Table 3, including basic statistics for each sample.

Since no scale of tolerance for contradiction exists in the literature, we reviewed key qualitative works on the subject (Graham 1986; Li 1998, 2012; Peng and Nisbett 1999) to get inspiration grounded in theory for framing a scale of tolerance for contradiction. A total of four items measuring tolerance for contradiction were thus developed and answered by respondents immediately after exposure to the two-sided ad: "Everything has both positive and negatives sides", "Good news can be changed to bad news", "Nothing is either black or white", and "I do not make a decision unless I carefully consider all sides of the story." The four items showed good reliability $(\alpha=0.83)$ and were aggregated to compare Chinese and American consumers. The findings show that Chinese consumers display a much firmer belief in YYB theory than Americans $\left(M_{\text {Easterners }}=5.45, M_{\text {Westerners }}=4.63 ; \mathrm{F}(1,421)\right.$ $=66.723, p<0.001)$, confirming a strong manipulation of YYB theory between Easterners and Westerners. 
Table 3. Psychometric Properties of the Measures, correlations, and ANOVA tests.

\begin{tabular}{|c|c|c|c|c|c|c|c|c|c|c|c|}
\hline \multirow{2}{*}{ Item per Construct } & \multicolumn{5}{|c|}{ Easterners $(n=227)$} & \multicolumn{5}{|c|}{ Westerners $(n=196)$} & \multirow[b]{2}{*}{$F$-Test (ANOVA) } \\
\hline & Mean & s.d. ${ }^{a}$ & $\alpha^{b}$ & $\mathrm{CR}^{\mathrm{c}}$ & $\operatorname{AVE}^{d}$ & Mean & s.d. ${ }^{a}$ & $\alpha^{b}$ & $\mathrm{CR}^{\mathrm{c}}$ & $\operatorname{AVE}^{\mathrm{d}}$ & \\
\hline 1. Tolerance for contradiction [ 1 "Strongly disagree" -7 "Strongly agree"] & 5.45 & 1.36 & \multirow[t]{5}{*}{0.82} & \multirow[t]{5}{*}{0.82} & \multirow[t]{5}{*}{0.53} & 4.63 & 1.01 & \multirow[t]{5}{*}{0.70} & \multirow[t]{5}{*}{0.70} & \multirow[t]{5}{*}{0.54} & \multirow[t]{5}{*}{$F(1,421)=66.723^{* * *}$} \\
\hline If there is good news, there will be bad news too & 5.16 & 1.40 & & & & 4.23 & 1.43 & & & & \\
\hline There is no absolute good and bad & 5.60 & 1.22 & & & & 4.48 & 1.73 & & & & \\
\hline Too much is as bad as too little & 5.31 & 1.37 & & & & 4.70 & 1.50 & & & & \\
\hline It's almost always good not to go to extremes, but rather to keep within a compass & 5.71 & 1.16 & & & & 5.11 & 1.16 & & & & \\
\hline 2. Credibility [ 1 "Strongly disagree"- 7 "Strongly agree"] & 4.82 & 1.41 & \multirow[t]{3}{*}{0.89} & \multirow[t]{3}{*}{0.90} & \multirow[t]{3}{*}{0.76} & 4.22 & 1.33 & \multirow[t]{3}{*}{0.92} & \multirow[t]{3}{*}{0.92} & \multirow[t]{3}{*}{0.80} & \multirow[t]{3}{*}{$F(1,421)=22.419^{* * *}$} \\
\hline In general, I trust the advertisement & 4.84 & 1.33 & & & & 4.29 & 1.40 & & & & \\
\hline In general, I feel properly guided by the advertisement & 4.74 & 1.48 & & & & 4.15 & 1.47 & & & & \\
\hline 3. Attitude towards the advertising [ 1 "Very unlikeable" -7 "Very likeable"] & 3.96 & 1.25 & \multirow[t]{3}{*}{0.90} & \multirow[t]{3}{*}{0.90} & \multirow[t]{3}{*}{0.81} & 3.65 & 1.44 & \multirow[t]{3}{*}{0.87} & \multirow[t]{3}{*}{0.87} & \multirow[t]{3}{*}{0.77} & \multirow[t]{3}{*}{$F(1,421)=5.807^{*}$} \\
\hline Bad/good & 4.05 & 1.26 & & & & 3.63 & 1.48 & & & & \\
\hline Negative/positive & 3.88 & 1.36 & & & & 3.67 & 1.57 & & & & \\
\hline 4. Attitude towards the brand [ 1 "Highly unfavourable" -7 "Highly favourable"] & 4.15 & 1.27 & \multirow[t]{3}{*}{0.80} & \multirow[t]{3}{*}{0.81} & \multirow[t]{3}{*}{0.69} & 3.84 & 1.58 & \multirow[t]{3}{*}{0.95} & \multirow[t]{3}{*}{0.95} & \multirow[t]{3}{*}{0.91} & \multirow[t]{3}{*}{$F(1,421)=4.926^{*}$} \\
\hline Bad/good & 4.16 & 1.47 & & & & 3.84 & 1.63 & & & & \\
\hline Negative/positive & 4.14 & 1.31 & & & & 3.84 & 1.62 & & & & \\
\hline 5. Purchase intentions [ 1 "Strongly disagree" -7 "Strongly agree"] & 4.28 & 1.36 & \multirow[t]{2}{*}{0.92} & 0.92 & 0.80 & 3.86 & 1.69 & 0.96 & 0.96 & 0.89 & $F(1,421)=8.011 * *$ \\
\hline If I need a cold medicine, I am very likely to have this product & 4.30 & 1.49 & & & & 3.95 & 1.75 & & & & \\
\hline
\end{tabular}

${ }^{\text {a }}$ Standard deviation; ${ }^{\mathrm{b}}$ Cronbach's alpha; ${ }^{\mathrm{c}}$ Coefficient of Reliability; ${ }^{\mathrm{d}}$ Average Variance Extracted; ${ }^{*} p<0.05,{ }^{* *} p<0.01,{ }^{* * *} p<0.001$. 


\section{Results}

\subsection{Validity and Reliability}

To ensure that all items load on their intended factor and do not cross-load on other factors, we performed an Exploratory Factor Analysis (EFA) using the ML method and Oblimin direct rotation technique. As expected, the EFA yielded the expected five-factor model, explaining $83 \%$ of the total variance, for a Kaiser-Meyer-Olkin index of 0.918 and a significant Bartlett's test of sphericity $\left(\chi^{2}=2449.82, d f=91, p<0.001\right)$. All items loaded on their intended factor, and none displayed cross-loadings or factor loadings below the critical value of 0.5 . The EFA also enabled the computation of the item-total correlations, that is, the correlation between each item variable of a given construct and the total score of that construct. The item-total correlations are above the 0.5 threshold, suggesting preliminary evidence for internal consistency in all model constructs.

A Confirmatory Factor Analysis (CFA) involving all the scales in the model assessed measurement quality. Global fit results were satisfactory for the total $\left(\chi_{(67)}^{2}=119.49 ; \mathrm{NFI}=0.970\right.$; $\mathrm{NNFI}=0.982 ; \mathrm{CFI}=0.987 ; \mathrm{MFI}=0.940 ; \mathrm{SRMR}=0.040 ; \mathrm{RMSEA}=0.043[0.030,0.055]$, American $\left(\chi_{(67)}^{2}=86.60 ; \mathrm{NFI}=0.960 ; \mathrm{NNFI}=0.987 ; \mathrm{CFI}=0.991 ; \mathrm{MFI}=0.951 ; \mathrm{SRMR}=0.050\right.$; RMSEA $=0.039[0.000,0.060])$, and Chinese $\left(\chi_{(67)}^{2}=72.12 ; \mathrm{NFI}=0.967 ; \mathrm{NNFI}=0.997\right.$; $\mathrm{CFI}=0.998 ; \mathrm{MFI}=0.989 ; \mathrm{SRMR}=0.033 ;$ RMSEA $=0.036[0.000,0.056])$ datasets. All factor loadings were high, with factor loadings close to or above 0.6. Cronbach's alphas and Composite Reliability displayed satisfactory values, thus supporting reliability of the scales (see Table 3). In addition, the average variance extracted (AVE) values for all the scales were greater than 0.5 , further supporting convergent validity (see Table 4; Fornell and Larcker 1981).

Table 4. Correlation matrix.

\begin{tabular}{|c|c|c|c|c|c|c|c|c|c|c|}
\hline \multirow[b]{2}{*}{ Interconstruct correlations } & \multicolumn{5}{|c|}{ Easterners $(n=227)$} & \multicolumn{5}{|c|}{ Westerners $(n=196)$} \\
\hline & 1 & 2 & 3 & 4 & 5 & 1 & 2 & 3 & 4 & 5 \\
\hline 1. Tolerance for contradiction & 0.73 & & & & & 0.73 & & & & \\
\hline 2. Credibility & 0.72 & 0.87 & & & & 0.71 & 0.89 & & & \\
\hline 3. Attitude towards the advertising & 0.28 & 0.16 & 0.90 & & & 0.40 & 0.41 & 0.88 & & \\
\hline 4. Attitude towards the brand & 0.72 & 0.67 & 0.30 & 0.83 & & 0.04 & 0.12 & 0.11 & 0.95 & \\
\hline 5. Purchase intentions & 0.70 & 0.53 & 0.23 & 0.72 & 0.89 & 0.38 & 0.15 & 0.04 & 0.25 & 0.94 \\
\hline
\end{tabular}

Note: the bold numbers represent the AVE square roots.

Discriminant validity was assessed in two ways. First, discriminant validity was assessed by comparing the interfactor correlations to the square root of their corresponding AVE. As shown in Table 4, the shared variance between constructs remained below the square root of the corresponding AVE, ensuring discriminant validity (Fornell and Larcker 1981). Second, we used a pairwise restriction of models (Anderson and Gerbing 1988). The correlation between each pair of factors was fixed to equal 1.0, and the significance of the chi-squared change was tested. All the chi-squared changes were significant, showing a better model fit for the model without these restrictions (see Table 5).

We randomized questions in the questionnaire to rule out common methods bias (Podsakoff et al. 2003). For example, attitude items were inserted in the credibility scale and vice versa. We also used Harman's single-factor test and found that a single factor yields a considerably worse model fit across the total, indicating that no single factor accounts for the covariance between the measures. In addition, we also used the common marker variable technique to rule out the common method variance. We used the post-hoc method of adding an unmeasured marker variable on the four dependent variables of the model (i.e., CRED, ATT_AD, ATT_BR, INT) to ensure that a method factor does not distort excessively the variance of those variables. The findings suggested that the influence of that marker variable was insignificant for CRED $(p=0.121)$, ATT_AD $(p=0.166)$, ATT_BR $(p=0.083)$, and INT $(p=0.803)$. Moreover, the difference between the R-square of dependent 
variables with and without marker variables was slight (i.e., less than $10 \%$ ). Thus, common method variance is not an issue.

Table 5. Chi-squared difference tests for assessing discriminant validity.

\begin{tabular}{ccccccc}
\hline & \multicolumn{2}{c}{ TOTAL $(n=423)$} & \multicolumn{2}{c}{ WESTERNERS $(n=196)$} & \multicolumn{2}{c}{ EASTERNERS $(n=227)$} \\
\hline & $\chi^{2}$ Value & $\Delta \chi^{2}(d f=1)$ & $\chi^{2}$ Value & $\Delta \chi^{2}(d f=1)$ & $\chi^{2}$ Value & $\Delta \chi^{2}(d f=1)$ \\
\hline Unconstrained model $(d f=67)$ & 119.49 & - & 86.60 & - & 72.12 \\
Constrained model $(d f=66)$ & & & & & \\
TFC-ATTAD & 178.01 & $58.52^{* * *}$ & 135.70 & $49.10^{* * *}$ & 101.37 & $29.25^{* * *}$ \\
TFC-ATTBR & 174.24 & $54.75^{* * *}$ & 136.70 & $50.10^{* * *}$ & 97.26 & $25.14^{* * *}$ \\
TFC-CRED & 182.78 & $63.29^{* * *}$ & 128.88 & $42.28^{* * *}$ & 88.72 & $16.60^{* * *}$ \\
TFC-INT & 160.32 & $40.83^{* * *}$ & 127.25 & $40.65^{* * *}$ & 90.27 & $18.15^{* * *}$ \\
ATTAD-ATTBR & 132.87 & $13.38^{* * *}$ & 122.47 & $35.87^{* * *}$ & 86.59 & $14.47^{* * *}$ \\
ATTAD-CRED & 135.28 & $15.79^{* * *}$ & 90.73 & $4.13^{*}$ & 87.80 & $15.68^{* * *}$ \\
ATTAD-INT & 134.96 & $15.47^{* * *}$ & 103.31 & $16.71^{* * *}$ & 87.48 & $15.36^{* * *}$ \\
ATTBR-CRED & 134.25 & $14.76^{* * *}$ & 99.16 & $12.56^{* * *}$ & 86.70 & $14.58^{* * *}$ \\
ATTBR-INT & 150.64 & $31.15^{* * *}$ & 139.27 & $52.67^{* * *}$ & 86.86 & $14.74^{* * *}$ \\
CRED-INT & 136.55 & $17.06^{* * *}$ & 98.94 & $12.34^{* * *}$ & 81.03 & $8.91^{* *}$ \\
\hline
\end{tabular}

Notes: $d f=$ degrees of freedom; ATTAD = attitude towards advertising; ATTBR = attitude toward the brand; CRED = credibility; INT = purchase intentions. ${ }^{*} p<0.05 .{ }^{* *} p<0.01 .{ }^{* * *} p<0.001$.

\subsection{Analysis and Results \\ 4.2.1. ANOVA}

We conducted ANOVAs between the two cultural groups (American vs. Chinese) on (a) tolerance for contradiction, (b) credibility, (c) attitude towards the ad, (d) attitude towards the brand, and (e) purchase intentions.

Chinese participants scored significantly higher on tolerance for contradiction than Americans (M_Easterners $=5.45$, M_Westerners $=4.63 ; \mathrm{F}(1,421)=66.723, p<0.001$ ), showing strong support for H1. Furthermore, similar results on tolerance for contradiction were obtained when taking ethnicity (vs. cultural group) into account (M_(East Asians) $=5.25$, M_(North American) = 4.56; M_Europeans $=4.57 ; \mathrm{F}(2,420)=54.62, p<0.001)$. Scheffe's post hoc test revealed that North Americans and Europeans do not differ regarding tolerance for contradiction $(p=0.998)$. Still, East Asians form a different sub-group and contrasted Western ethnicities in terms of the tolerance for contradiction $(p<0.001)$. Chi-squared tests further revealed that the cultural group indicated by respondents is indeed strongly associated with their ethnicity $\left(\chi^{2}=423, d f=2, p<0.001\right)$. In sum, respondents affiliating with East Asian (North American or European) ethnicity refer more to the Easterner (Westerner) cultural group, showing that the confounding effect of ethnicity is minimal and nonsignificant.

Chinese participants further showed higher credibility (M_Easterners $=4.82$, M_Westerners $=4.22 ; \mathrm{F}(1,421)=22.419, p<0.001)$, stronger attitude towards the advertising (M_Easterners $=3.96$, M_Westerners $=3.65 ; \mathrm{F}(1,421)=5.807, p<0.05)$, and attitude towards the brand (M_Easterners $=4.15$, M_Westerners $=3.84 ; \mathrm{F}(1,421)=4.926$, $p<0.05)$, as well as higher purchase intentions than Americans (M_Easterners $=4.28$, M_Westerners $=3.86 ; \mathrm{F}(1,421)=8.011, p<0.01)$. Although Americans had lower scores than Chinese, their ratings on credibility were higher than other variables, i.e., attitude towards the ad and brand. These preliminary findings indicate a process underlying the tolerance for contradiction-to-intention effect through the proposed mediating variables and that this effect differs between Chinese and American consumers. To test this process formally, we conducted separate mediation analyses for the total American and Chinese samples. The mediation analyses provide an insight into the validity of the hypotheses, the underpinning mediational processes, and the group differences regarding the hypothesized model. 


\subsubsection{Structural Model Estimation}

The structural model was then tested and yielded a good model fit $\left(\chi_{67}^{2}=119.49\right.$; $\mathrm{NFI}=0.970 ; \quad \mathrm{NNFI}=0.982 ; \quad \mathrm{CFI}=0.987 ; \quad \mathrm{MFI}=0.940 ; \quad \mathrm{SRMR}=0.040 ;$ RMSEA $=0.043$ [0.030, 0.055]), indicating a theoretically sound statistical model. The link between tolerance for contradiction and purchase intentions is nonsignificant, indicating a potential mediational effect.

\subsubsection{Hypotheses Testing}

A bootstrap mediation test with three mediators (Hayes and Preacher 2014) using PROCESS (January 2016 version) model 6 (3 mediators) was used to test the hypothesized relationships in the research model. Hayes' (2013) PROCESS macro for SPSS (model 6) is more powerful and rigorous than traditional multivariate procedures to test hypotheses. Besides, bootstrap is a more robust approach for testing mediation. It solves the non-normal sampling distribution problem produced from the indirect effect being a product of several parameters by generating an empirical sampling distribution of $a \times b$ (Hayes and Preacher 2014). The PROCESS macro also enables test mediation involving multiple mediators simultaneously, overcoming the necessity to conduct multiple separate mediation analyses, yielding less valid results (Hayes 2013). The estimates were based on 5000 resamples and a 95\% confidence level. Given satisfactory reliability indices for all constructs (see Table 3), we averaged the variables about each construct with each other to form single-item indices. The mediation analysis was performed in two steps. First, we examined the significance of the paths in the total dataset. Subsequently, we ran the mediation for Chinese and American consumers separately to identify the different model paths and mediation effects in both groups.

Total dataset. Table 6 shows that all direct hypothesized relationships are supported except one (i.e., H3b). These results collectively validate $\mathrm{H} 2 \mathrm{a}, \mathrm{H} 3 \mathrm{a}, \mathrm{H} 4 \mathrm{a}-\mathrm{b}, \mathrm{H} 5 \mathrm{a}, \mathrm{H} 6 \mathrm{a}, \mathrm{H} 7 \mathrm{a}$, and H8a. The lack of support for H3b could be attributed to the fact that the tolerance for contradiction will primarily influence (positively) the attitude toward the two-sided message for which balance will be needed to have a full appreciation. However, tolerance will not be as important in determining the attitude toward the brand that communicates that message. In other words, since the message rather than the brand conveys the contradiction, it follows that TFC is s stronger predictor of attitude toward the message than the brand since it is at the message level that reconciliation through rebalancing will be needed. Furthermore, following Hayes (2013), the nonsignificant effect of the independent variable, i.e., tolerance for contradiction, on the dependent variable, i.e., purchase intention, may suggest that the three proposed mediators (i.e., credibility, attitude toward the brand, and attitude toward the ad) fully mediate the relationship between tolerance for contradiction and intentions, thus lending a priori support for $\mathrm{H} 9 \mathrm{a}$.

Interestingly, age and gender tend to strengthen and significantly impact TFC on attitude toward the brand (H3b). Therefore, older respondents and women are more likely to hold positive attitudes toward the brand due to higher tolerance for contradiction. Education and ethnicity, however, do not seem to exert any significant impact on the positive relationships. 
Table 6. Results of the Bootstrap Mediation Tests a

\begin{tabular}{|c|c|c|c|c|c|c|c|c|c|c|c|}
\hline & \multirow[t]{2}{*}{ Path } & \multicolumn{3}{|c|}{ Total } & \multicolumn{3}{|c|}{ Westerners } & \multicolumn{3}{|c|}{ Easterners } & \multirow{2}{*}{$\begin{array}{c}\text { Difference Tes } \\
t \text {-value }\end{array}$} \\
\hline & & $\beta$ & s.e. & $t$-value & $\beta$ & s.e. & $t$-value & $\beta$ & s.e. & $t$-value & \\
\hline \multicolumn{12}{|l|}{ Direct effects } \\
\hline H3a: TFC $\rightarrow$ ATTAD & $a_{2}$ & 0.197 & 0.062 & $3.196^{* *}$ & 0.086 & 0.051 & 1.686 (ns) & 0.234 & 0.022 & $10.636^{* * *}$ & Н3c: $2.801^{* *}$ \\
\hline $\mathrm{H} 3 \mathrm{~b}: \mathrm{TFC} \rightarrow \mathrm{ATTBR}$ & $a_{3}$ & 0.037 & 0.044 & 0.853 (ns) & -0.087 & 0.074 & $-1.171(\mathrm{~ns})$ & 0.150 & 0.050 & $2.995^{* *}$ & H3c: 2.721 ** \\
\hline H4a: CRED $\rightarrow$ ATTAD & $d_{21}$ & 0.264 & 0.069 & $3.848^{* * *}$ & 0.044 & 0.099 & 0.445 (ns) & 0.492 & 0.081 & $6.082^{* * *}$ & H4c: $3.544^{* * *}$ \\
\hline H4b: CRED $\rightarrow$ ATTBR & $d_{31}$ & 0.289 & 0.064 & $4.513^{* * *}$ & 0.002 & 0.006 & 0.333 (ns) & 0.334 & 0.079 & $4.032^{* * *}$ & H4c: $3.905^{* * *}$ \\
\hline H5a: CRED $\rightarrow$ INT & $b_{1}$ & 0.186 & 0.043 & $4.294^{* * *}$ & 0.045 & 0.052 & 0.870 (ns) & 0.545 & 0.079 & $6.889^{* * *}$ & H5b: $5.115^{* * *}$ \\
\hline H6a: ATTAD $\rightarrow$ ATTBR & $d_{32}$ & 0.816 & 0.032 & $25.602 * * *$ & 0.719 & 0.041 & $17.669^{* * *}$ & 0.889 & 0.046 & $19.389^{* * *}$ & $2.728 * *$ \\
\hline H7a: ATTAD $\rightarrow$ INT & $b_{2}$ & 0.223 & 0.054 & $4.166^{* * *}$ & 0.116 & 0.007 & $16.571^{* * *}$ & 0.133 & 0.005 & $26.048^{* * *}$ & $2.002 *$ \\
\hline H8a: ATTBR $\rightarrow$ INT & $b_{3}$ & 0.617 & 0.052 & $11.725^{* * *}$ & 0.289 & 0.080 & $3.307^{* * *}$ & 0.843 & 0.058 & $14.494^{* * *}$ & $5.697 * * *$ \\
\hline \multirow{2}{*}{ H9a: TFC $\rightarrow$ INT } & C & 0.075 & 0.039 & 1.902 (ns) & 0.069 & 0.057 & 1.210 (ns) & 0.023 & 0.062 & 0.368 (ns) & 0.541 (ns) \\
\hline & & $\beta$ & s.e. & C.I. & $\beta$ & s.e. & C.I. & $\beta$ & s.e. & C.I. & $t$-value \\
\hline \multicolumn{12}{|l|}{ Indirect effects } \\
\hline H9a: Total indirect effect & $c^{\prime}$ & 0.188 & 0.062 & $(0.066,0.315)$ & 0.006 & 0.121 & $(-0.230,0.243)(\mathrm{ns})$ & 0.322 & 0.068 & $(0.198,0.462)$ & H6b-c: $2.362 *$ \\
\hline $\mathrm{TFC} \rightarrow \mathrm{CRED} \rightarrow \mathrm{INT}$ & $a_{1} b_{1}$ & 0.044 & 0.018 & $(0.016,0.086)$ & 0.010 & 0.016 & $(-0.008,0.064)(\mathrm{ns})$ & 0.031 & 0.020 & $(0.003,0.086)$ & 0.805 (ns) \\
\hline $\mathrm{TFC} \rightarrow \mathrm{ATTAD} \rightarrow \mathrm{INT}$ & $a_{2} b_{2}$ & 0.002 & 0.010 & $(-0.016,0.022)(\mathrm{ns})$ & 0.005 & 0.009 & $(-0.005,0.034)(\mathrm{ns})$ & 0.081 & 0.034 & $(0.024,0.158)$ & $2.029 *$ \\
\hline $\mathrm{TFC} \rightarrow \mathrm{ATTBR} \rightarrow \mathrm{INT}$ & $a_{3} b_{3}$ & 0.023 & 0.027 & $(-0.031,0.076)(\mathrm{ns})$ & -0.074 & 0.060 & $(-0.188,0.051)(\mathrm{ns})$ & 0.043 & 0.018 & $(0.015,0.088)$ & $1.988 *$ \\
\hline $\mathrm{TFC} \rightarrow \mathrm{CRED} \rightarrow \mathrm{ATTAD} \rightarrow \mathrm{INT}$ & $a_{1} d_{21} b_{2}$ & 0.010 & 0.005 & $(0.003,0.023)$ & 0.000 & 0.001 & $(-0.000,0.006)(\mathrm{ns})$ & 0.063 & 0.025 & $(0.024,0.126)$ & $2.345^{*}$ \\
\hline $\mathrm{TFC} \rightarrow \mathrm{CRED} \rightarrow \mathrm{ATTBR} \rightarrow \mathrm{INT}$ & $a_{1} d_{31} b_{3}$ & 0.002 & 0.003 & $(-0.002,0.009)(\mathrm{ns})$ & -0.001 & 0.002 & $(-0.010,0.001)(\mathrm{ns})$ & 0.026 & 0.012 & $(0.008,0.057)$ & $2.074^{*}$ \\
\hline $\mathrm{TFC} \rightarrow \mathrm{ATTAD} \rightarrow \mathrm{ATTBR} \rightarrow \mathrm{INT}$ & $a_{2} d_{32} b_{3}$ & 0.099 & 0.031 & $(0.043,0.165)$ & 0.064 & 0.077 & $(-0.084,0.221)(\mathrm{ns})$ & 0.049 & 0.022 & $(0.015,0.104)$ & 0.200 (ns) \\
\hline $\mathrm{TFC} \rightarrow \mathrm{CRED} \rightarrow \mathrm{ATTAD} \rightarrow \mathrm{ATTBR} \rightarrow \mathrm{INT}$ & $a_{1} d_{21} d_{32} b_{3}$ & 0.009 & 0.004 & $(0.003,0.020)$ & 0.001 & 0.003 & $(-0.001,0.012)(\mathrm{ns})$ & 0.029 & 0.012 & $(0.010,0.064)$ & $2.124 *$ \\
\hline
\end{tabular}


Table 6. Cont.

\begin{tabular}{|c|c|c|c|c|c|c|c|c|c|c|c|}
\hline & \multirow[t]{2}{*}{ Path } & \multicolumn{3}{|c|}{ Total } & \multicolumn{3}{|c|}{ Westerners } & \multicolumn{3}{|c|}{ Easterners } & \multirow[t]{2}{*}{ Difference Test } \\
\hline & & $\beta$ & s.e. & $t$-value & $\beta$ & s.e. & $t$-value & $\beta$ & s.e. & $t$-value & \\
\hline \multicolumn{12}{|l|}{ Total effect } \\
\hline \multicolumn{12}{|l|}{ Model } \\
\hline & $F$-value & & & $403.64^{* * *}$ & & & $367.99 * * *$ & & & $215.21^{* * *}$ & \\
\hline & $d f 1$ & & & 4 & & & 4 & & & 4 & \\
\hline & $d f 2$ & & & 418 & & & 191 & & & 222 & \\
\hline & Adj. $R^{2}$ & & & 0.714 & & & 0.791 & & & 0.713 & \\
\hline
\end{tabular}

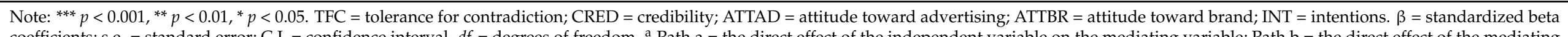

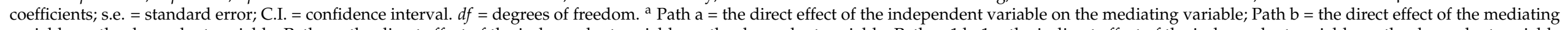

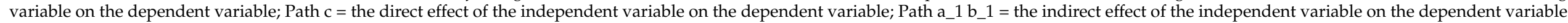

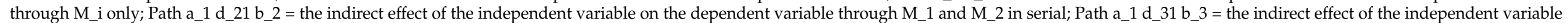

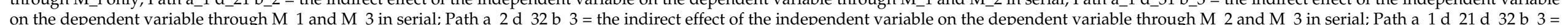

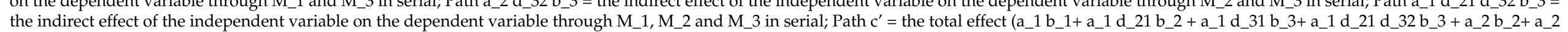
d_ 32 b_3 $+a \_3 b \_3+c$ ) 
Group differences. Table 6 and Figure 5 show that all the hypothesized direct relationships (i.e., H3a-b, H4a-b, H5a, H6a, H7a, and H8b) are significant in the Chinese group. These results contrast sharply with those of the American group, where almost all of the hypothesized direct relationships (i.e., H3a-b, H4a-b, and H5a) are not significant except for H6a, H7a, and H8a (see Table 6 and Figure 6). As expected, the hypothesized paths are significantly stronger in the Chinese dataset than in the American one, providing support for $\mathrm{H} 2 \mathrm{~b}, \mathrm{H} 3 \mathrm{c}, \mathrm{H} 4 \mathrm{c}, \mathrm{H} 5 \mathrm{~b}, \mathrm{H} 6 \mathrm{~b}, \mathrm{H} 7 \mathrm{~b}$, and $\mathrm{H} 8 \mathrm{~b}$. The tolerance for contradiction-intention link (H9a) is nonsignificant for both Westerners $(\beta=0.069, \mathrm{t}=1.210, \mathrm{n} . \mathrm{s}$. $)$ and Easterners $(\beta=0.023, t=0.368$, n.s. $)$, suggesting potentially a full mediation effect of the mediators on that relationship.

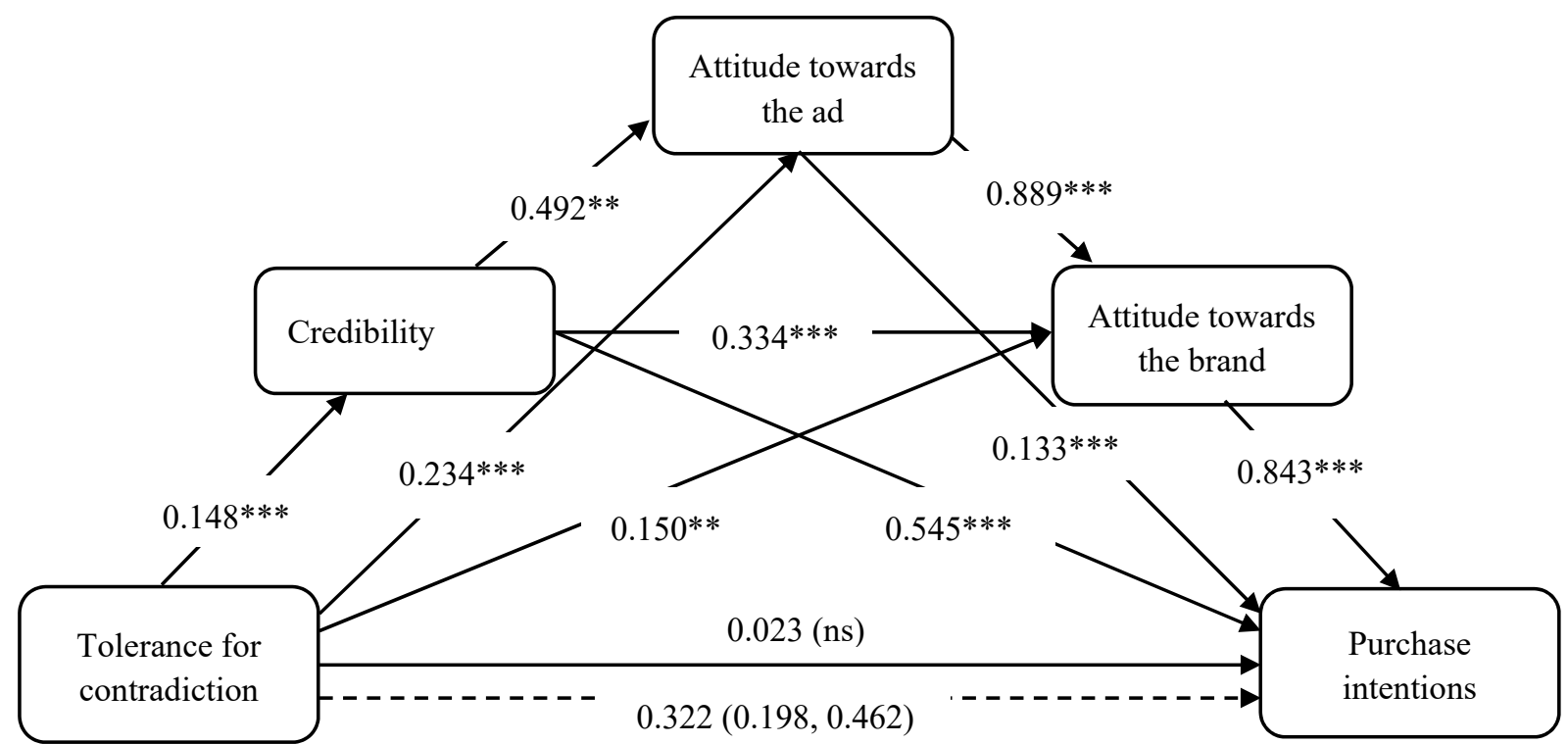

Figure 5. Conceptual model with results for Easterners. Note: ${ }^{* *} p<0.01,{ }^{* * *} p<0.001$.

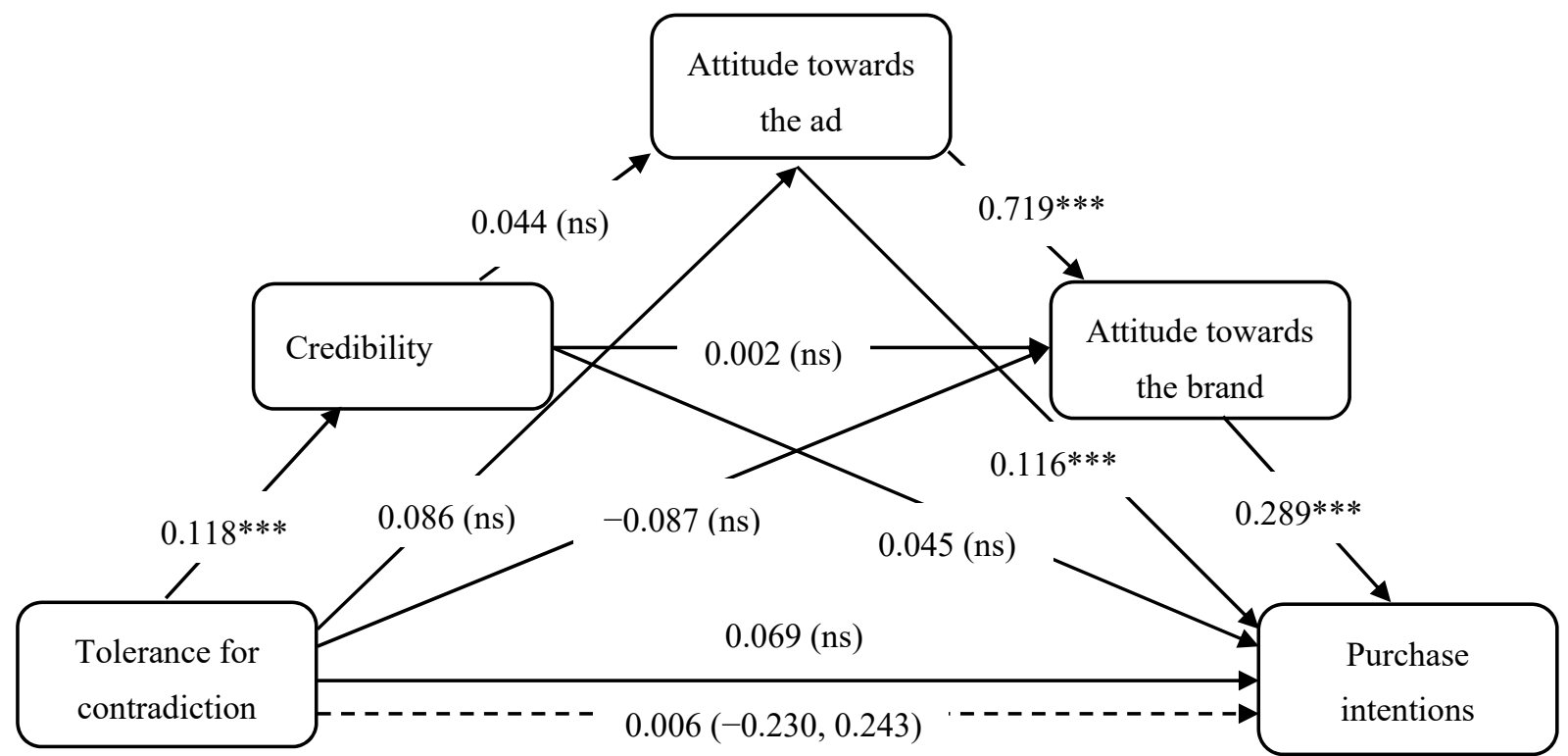

Figure 6. Conceptual model with results for Westerners. Note: ${ }^{* *} p<0.001$.

As mentioned earlier, since TFC influences females to a greater degree in their attitude toward the brand and that the Chinese sample consists of comparatively more women (while the US sample contains more men), this might explain the support for H3b found 
in the Chinese sample. Interestingly, in the Chinese sample, age, gender, education, and ethnicity were also found to influence TFC and credibility more strongly (H2a). This means that in the Chinese sample, older respondents, women, the highly educated, and ethnic Asians are more likely to see their TFC influence the perceived credibility of the ad. Again, since more women comprise the Chinese dataset, this might explain the support for $\mathrm{H} 2 \mathrm{a}$. However, such effects of covariates were not found for other hypotheses on which the US and Chinese samples differ.

\subsubsection{Mediation Analysis}

We proceeded with the testing of the direct effect of the independent variable (i.e., tolerance for contradiction) on the mediating variables (i.e., credibility, attitude towards the ad, and attitude towards the brand) (paths a), the direct effect of the mediating variables on the dependent variable (i.e., purchase intentions) (paths b), the direct effect of the independent variable on the dependent variable (path c), the total effect of the independent variable on the dependent variable (path $c^{\prime}$ ) and a series of indirect effects (see Table 6).

Total dataset. The results suggest an indirect effect of the tolerance for contradiction on purchase intentions, mainly through attitudes toward the advertisement and attitude toward the brand, on the one hand, and credibility on the other hand.

Group differences. In the American group, no significant indirect effect was found $(\beta=0.006,[-0.230,0.243])$, suggesting an absence of mediation of tolerance for contradiction on purchase intentions through the hypothesized mediator variables (see Table 6 and Figure 6). In contrast, the indirect effect was significant $(\beta=0.322,[0.198,0.462])$ in the Chinese group (see Table 6 and Figure 5), supporting H9b. Moreover, the nonsignificant impact of TFC on intentions $(\beta=0.069, \mathrm{~ns})$ suggests a full mediation, meaning that the effect of tolerance for contradiction on purchase intentions is fully explained by credibility, the attitude toward the ad, and the attitude toward the brand. Attitude toward the ad seems the most critical mediator in that full mediation, since the mediational links involving attitude toward the ad account for $69.8 \%$ of the total indirect effect, which is higher than credibility $(46.3 \%)$ and attitude towards the brand $(45.6 \%)$. These results suggest that firstly, attitude toward the ad, and secondly, credibility and attitude toward the brand explain the positive effect of the tolerance for contradiction on the purchase intentions of Chinese participants.

\section{Discussion}

The results show that the place of residence is highly correlated with both ethnicity and differences in tolerance for contradiction. While Westerners (Easterners) affiliate predominantly with North Americans and European ethnicities (Chinese ethnicity), they score significantly lower (higher) on the tolerance for contradiction. From past literature (e.g., Fang 2012; Li 2012), both Easterners and Westerners do therefore meaningfully and significantly differ in terms of the tolerance for contradiction, with Easterners (Westerners) being more (less) prone to tolerance of inconsistency and resolution among contrasts than Westerners (Easterners) (Fang and Faure 2011).

Further, these results suggest validity for our assumption that the relationship between the tolerance for contradiction and purchase intentions is mainly explained by the mediating variables of attitude toward the ad and credibility. As hypothesized, American and Chinese consumers differed. Whether direct or indirect, no effect could be observed between the tolerance for contradiction and purchase intentions for American consumers. In contrast, for Chinese consumers, attitude toward the ad and credibility and attitude toward the brand are significant mediating variables.

More generally, these results suggest an adverse effect that is not counterbalanced by a predisposition for nuanced thinking, i.e., according to the YYB framework, a higher tolerance for contradiction (Li 1998; Zolfani et al. 2013) does not re-equilibrate that imbalanced relationship. In sum, the tolerance for contradiction mitigates that negative causal chain 
by infusing an enhanced sense of acceptance of contradictions and counter-intuitiveness (Li 1998, 2012; Peng and Nisbett 1999).

Testing the hypothesized model on the total dataset suggests that the tolerance for contradiction impacts credibility and attitude towards the ad, but not the attitude towards the brand, potentially because of the American subset. Had cultural differences, or to the least, differences in tolerance for contradiction, not been controlled for, this finding would have appeared as mixed and confusing.

Consistent with some past research (e.g., Uribe et al. 2016; Chen 2016; Li et al. 2020; Huertas and Hanna 2020), we find that two-sided ads are more persuasive because they increase source credibility. Furthermore, we also find that this link is stronger for Chinese than American consumers, suggesting a higher perception of source credibility for individuals with a higher tolerance for contradiction. The two-sided advertising format displayed by the brand is thus more "fitting" with Chinese consumers' holistic or balanced thinking style "involving an orientation to the context or field as a whole, including attention to relationships between a focal object and the field, and a preference for explaining and predicting events based on such relationships" (Nisbett et al. 2001, p. 293). In contrast to Westerners' piecemeal, discrete, or discontinuous thinking style-where product utility and functionality impact attitude towards the brand (Monga and John 2004)_Easterners' enhanced attitude toward the advertising and the brand derives directly from their higher tolerance for contradiction.

We find no mediational effect of the tolerance for contradiction on purchase intentions through the three proposed mediators for American consumers. In contrast, we find the full mediation and strong indirect effect for Chinese, explaining the tolerance for contradiction-purchase intention relationship. Moreover, while attitude toward the ad seems to play an important role, attitude toward the brand and credibility also equally explain a significant share of the relationship. Therefore, compared to Westerners, Eastern consumers' higher purchase intentions result primarily from an enhanced attitude toward the ad and enhanced credibility and attitude towards the brand. All of these effects are spurred by balancing needs.

In conclusion, Chinese consumers' higher propensity to relativize, i.e., tolerance for contradiction, although not directly related to purchasing intentions, infuses an improved attitude toward the ad, increased credibility and an improved attitude toward the brand, which reverberate both directly and indirectly, on the increase in purchase intentions.

\section{Implications}

\subsection{Theoretical Implications}

Past research on the effectiveness of two-sided messages mostly originates from Western countries and showed inconsistent results (Kamins and Marks 1987; Trifts and Häubl 2003; Eisend 2007, 2008, 2010; Cornelis et al. 2012, 2015; Pizzutti et al. 2016). These perspectives are also limited in an international marketing context and especially in settings involving Eastern-Western relationships. We propose that the YYB theory (Li 1998, 2012; Nisbett et al. 2001; Peng and Nisbett 1999; Zolfani et al. 2013) is an essential framework for understanding the effectiveness of two-sided messages, and its inclusion enables us to comprehend the mechanism underlying two-sided message persuasiveness. In contrast to previous research, which used, among others, the Elaboration Likelihood Model (Kao 2011; Qi et al. 2010a, 2010b), we use the YYB (Yin-Yang Balancing) framework to focus on explaining Eastern consumers' preference for two-sided messages over Westerners'. Overall, this study showed that the tolerance for contradiction from the East Asian philosophical framework YYB theory infuses a critical perspective to understand the persuasiveness of two-sided ads. More specifically, differences in tolerance for contradiction exert a determining effect on two-sided ads' persuasiveness.

This study hints at several differences between Easterners and Westerners in terms of direct and mediational effects, and these differences may, in turn, increase our under- 
standing of two-sided ad effectiveness. This study has three main implications that could explain Westerners' lower propensity to purchase a product advertised in a two-sided ad.

First, the Westerners' purchase intentions culminate from their attitude towards the ad. Therefore, a negative attitude towards the ad is not balanced and may consequently lower purchase intentions. Our finding is consistent with past studies which showed that Westerners are more akin to a structural (vs. holistic) frame of thinking where ad and product details are analyzed in a piecemeal sequence, which leads to attitude formation (Choi et al. 1997; Nisbett et al. 2001; Sugitani 2014). Furthermore, our results show that this sequence of attitude formation is not influenced by a "balanced" approach to thinking. Therefore, attitudes determine purchase likelihood in an independent and isolated manner so that the tolerance does not compensate an immediate unfavourable response to the ad for ambiguity, paradox, and inconsistency, typically induced by the tolerance for contradiction (Fang 2012).

Second, past research showed that Westerners, compared to Easterners, are typically more skeptical of brands (MacInnis et al. 2014; Sugitani 2014). Therefore, if a negative attitude towards the ad is not counterbalanced by a more holistic frame of thinking, that negative attitude will reverberate towards the brand. The latter will subsequently decrease the purchase likelihood for Westerners.

Third, we advance past literature that showed that two-sided ads enhance credibility by demonstrating that tolerance for contradiction is positively related to credibility. In addition to credibility, enhanced attitude toward the ad is even more critical since attitude toward the brand is strongly influenced by attitude toward the ad that strongly influences purchase intentions. Thus, lower tolerance levels for contradiction in Western datasets may account for the mixed findings regarding two-sided messages' persuasiveness in the Western literature.

The results of this study also have several implications for understanding Easterners' higher likelihood to purchase consumer products advertised by two-sided ads. The results show that the YYB framework lies at the core of that enhanced likelihood.

First, contrary to Westerners, Easterners' attitudes towards the ad, attitudes toward the brand, and credibility are significantly influenced by their tolerance for contradiction. Their higher tolerance and non-denial of contradiction enable them to resolve better attitude formation conflicts resulting from two opposites, typically found in two-sided messages (Li 2012). This aspect is crucial since the tolerance for contradiction does not directly impact purchase intention. Instead, through multiple mediational processes involving attitude formation and credibility perception, the tolerance for contradiction may ultimately affect purchase intentions.

Second, compared to attitudes, credibility appears as a secondary conveyor of the tolerance for contradiction into purchase intentions. Interpretation of the results helps provide a more in-depth explanation of the role of attitude. Attitude toward the ad is the mediator that is the best explanatory variable of the relationship between the tolerance for contradiction and purchase intentions. This may be because contrarily to Westerners who may form better attitudes toward the ad, primarily a result of the enhanced transparency about product functionality (Sugitani 2014), typically conveyed by the two-sided message, Easterners may develop a better attitude toward the ad as a result of their exposure to a message which they perceive as "befitting" with their thinking frame, thus being more credible. Enhanced attitude toward the ad for Easterners is not the result of enhanced credibility about product quality or features (as for Westerners), but their inherent preference for a message that espouses their thinking style and that they find credible because it corresponds to their cultural predispositions.

Due to the inconsistent results observed in the literature regarding two-sided message effectiveness, a whole stream of research has started investigating the specific conditions under which two-sidedness yields superior results to one-sidedness. We complement this stream which showed that two-sided messages are more effective for individuals with a high need for cognition (NFC) (Qi et al. 2010a, 2010b; Kao 2011) and high self- 
confidence (Huertas and Hanna 2020) for search goods as compared to experience goods (Chen 2016; Li et al. 2020), or when the source of the message is perceived to be an expert (Chen 2016), by further showing that two-sided messages are more effective for individuals with a higher tolerance for contradiction. According to YYB, that characteristic squarely fits with Easterners who show a higher tolerance for contradiction, and by extrapolation, two-sided messages are more effective for Easterners, and Chinese in particular, than for Westerners and Americans in particular. The results provided solid empirical evidence for the predictions and provided an additional level of explanation as to the conditions in which two-sidedness is more effective, at least in driving purchase intentions.

This study emphasizes that the key construct of tolerance for contradiction lies at the root of the differences in reaction to two-sided ads between Easterners and Westerners. While the differences in tolerance for contradiction have been found between both populations, varying levels of tolerance for contradiction may also exist among Easterners and Westerners themselves. For example, some Westerners may have a higher tolerance for contradiction than Westerners' general population and thus react more positively to two-sided ads, as suggested by this study. Therefore, this research sheds some light on one additional variable, namely the tolerance for contradiction, that is worth being considered in an attempt to understand the effectiveness of two-sided advertising.

\subsection{Managerial Implications}

We find that consumers with a higher tolerance for contradiction, such as Easterners, will be more receptive to advertising featuring contradictory or counterintuitive messages, such as two-sided ads. On the other hand, consumers with a lower tolerance for contradiction, such as Westerners, will be less receptive to such framings. Although there might always be exceptions in both populations, in general, advertising managers may favour two-sided advertising when addressing Eastern consumers but prefer one-sided messages or even comparative advertising, emphasizing product functionality (e.g., Putrevu and Lord 1994) when addressing Westerners. Past research showed that among East Asian consumers, two-sided ads are especially effective with expert consumers compared to novice ones (Chen 2016). Likewise, Western consumers may consider two-sided ads more positively when they are experts.

From a broader perspective, it has been more than 170 years now that a process of East-Meeting-West has been engaged, but this trend has accelerated over the last 50 years (Lavagnino 2017). Since capitalism was blended into the Chinese social communist system (toward the 1970s), one-sided messages have become a norm in the Chinese cultural and commercial landscape. Meanwhile, two-sided messages remained marginal in the West, and their impact mixed, as amply discussed in this paper. Yet, as the world becomes even more interactive and integrated, the principles of YYB are likely to be more prevalent in advertising shown to Westerners. Using two-sided ads and messages recurrently could spur Westerners' sense of reconciling opposites instead of considering them as opposites. As they become a more common part of their (advertising) culture, Westerners might eventually develop a stronger connection to those messages, and their impact will probably be less mixed. Such hypotheses will need to be tested more rigorously in future practitioner and/or academic research.

\section{Limitations and Future Research Avenues}

In the present study, we used a priming cue, an over-the-counter cough medicine, which confluences pharmaceuticals and fast-moving consumer goods. Further, consumers' preferences for this type of product may differ by their origin (cf. Zhou and Belk 2004). It will be useful for future research to test with a variety of different product categories.

As this study was designed to compare the countries, it overlooked individual differences within each culture, especially for the US with a heterogeneous population. Similarly, differences in tolerance for contradiction could also be found within specific cultures, since some people may have a higher tolerance for contradiction than others, which may further 
explain the mixed findings regarding two-sided ads that were reported in the literature (e.g., Kao 2011). Future research should investigate this possibility.

Additionally, since past research found that two-sided messages were effective with East Asians in general and expert ones in particular (Chen 2016), future studies could further investigate whether the relative lack of effectiveness of two-sided ads among Westerners as compared to Easterners is attenuated among specific consumer categories, namely expert versus novice groups.

Participants in this study were exposed to a print advertisement of a hypothetical product for an unknown brand. We decided not to create a persuasion situation in which participants were familiar with the product, which could bias the results. However, under conditions of an unaware audience, some respondents might choose the medium-scale position in the questionnaire that harbors a neutral opinion rather than expressing highly favorable or unfavorable opinions about the product or advertisement, which may have dampened the effects of the message sidedness. Therefore, we checked the distribution of results about each group and found that all variables displayed excellent skewness and kurtosis values, strictly below the absolute value of 1 , either -1 or 1 (Nunnally and Bernstein 1994) for each item and each variable index. Therefore, we believe that our approach did not bias the effects.

Author Contributions: Conceptualization, E.S., F.K. and M.-S.J.; methodology, E.S. and M.-S.J.; software, M.E.; validation, M.E., E.S. and M.-S.J.; formal analysis, M.E.; investigation, M.E., E.S., M.-S.J.; resources, E.S. and M.-S.J.; data curation, M.E., E.S.; writing—original draft preparation, M.E.; writing-review and editing, E.S., F.K., M.-S.J.; visualization, M.E.; supervision, E.S., F.K., M.-S.J.; project administration, E.S. All authors have read and agreed to the published version of the manuscript.

Funding: This research received no external funding.

Institutional Review Board Statement: The study posed minimal threat to the respondents and conducted according to the guidelines of the Declaration of Helsinki.

Informed Consent Statement: Informed consent was obtained from all subjects involved in the study.

Data Availability Statement: The data are available from authors upon request.

Acknowledgments: We are thankful to an anonymous reviewer for the suggestion and for the references.

Conflicts of Interest: The authors declare no conflict of interest.

\section{References}

Ajzen, Icek. 1991. The theory of planned behavior. Organizational Behavior and Human Decision Processes 50: 179-211. [CrossRef]

Allen, Mike. 1991. Meta-analysis comparing the persuasiveness of one-sided and two-sided messages. Western Journal of Speech Communication 55: 390-404. [CrossRef]

Anderson, James C., and David W. Gerbing. 1988. Structural equation modeling in practice: A review and recommended two-step approach. Psychological Bulletin 103: 411-23. [CrossRef]

Belch, George E. 1983. The effects of message modality on one-and two-sided advertising messages. ACR North American Advances Advances in Consumer Research 10: 21-26.

Beltramini, Richard F. 1988. Perceived believability of warning label information presented in cigarette advertising. Journal of Advertising 17: 26-32. [CrossRef]

Cameron, Kim S., and Robert E. Quinn. 1988. Organizational Paradox and Transformation. In Paradox and Transformation: Toward a Theory of Change in Organization and Management. Edited by Quinn Robert E and Kim S. Cameron. Cambridge: Ballinger, pp. 1-18.

Campos, Anthony. 2018. Two-Sided Messaging: A Quantitative Analysis of Humor-ous Warmth Impacting Message Attitude, Source Credibility, and Persuasion. Ph.D. thesis, Northcentral University, Scottsdale, AZ, USA.

Chebat, Jean-Charles, Mathieu Charlebois, and Claire Gélinas-Chebat. 2001. What makes open vs. closed conclusion advertisements more persuasive? The moderating role of prior knowledge and involvement. Journal of Business Research 53: 93-102. [CrossRef]

Chen, Ming-Jer. 2002. Transcending paradox: The Chinese "middle way" perspective. Asia Pacific Journal of Management 19: 179-99. [CrossRef]

Chen, Ming-Jer. 2008. Reconceptualizing the competition-Cooperation relationship: A transparadox perspective. Journal of Management Inquiry 17: 288-304. [CrossRef] 
Chen, Ming-Yi. 2016. Can two-sided messages increase the helpfulness of online reviews? Online Information Review 40: 316-32. [CrossRef]

Chin, Tachia, Chris Rowley, Gordon Redding, and Shouyang Wang. 2018. Chinese strategic thinking on competitive conflict: Insights from Yin-Yang harmony cognition. International. Journal of Conflict Management 29: 683-704. [CrossRef]

Choi, Incheol, Richard E. Nisbett, and Edward E. Smith. 1997. Culture, category salience, and inductive reasoning. Cognition 65: 15-32. [CrossRef]

Cooper, Jean C. 1990. Taoism: The Way of the Mystic, Reprint Edition. Welling Borough: The Aquarian Press.

Cornelis, Erlinde, Verolien Cauberghe, and Patrick De Pelsmacker. 2012. The Impact of Message Sidedness on Adolescents' Binge Drinking Intentions after Peer Pres-sure: The Moderating Role of Issue Involvement. Advances in Advertising Research. Wiesbaden: Gabler Verlag, vol. III, pp. 69-79.

Cornelis, Erlinde, Veroline Cauberghe, and Patrick De Pelsmacker. 2014. The inoculating effect of message sidedness on adolescents' binge drinking intentions: The moderating role of issue involvement. Journal of Drug Issues 44: 254-68. [CrossRef]

Cornelis, Erlinde, Verolien Cauberghe, and Patrick De Pelsmacker. 2015. The credibility of refutation in two-sided anti-drug messages. Journal of Social Marketing 5: 241-57. [CrossRef]

Cornelis, Erlinde, Nico Heuvinck, and Anuja Majmundar. 2020. The ambivalence story: Using refutation to counter the negative effects of ambivalence in two-sided messages. International Journal of Advertising 39: 410-32. [CrossRef]

Crowley, Ayn E., and Wayne D. Hoyer. 1994. An integrative framework for under-standing two-sided persuasion. Journal of Consumer Research 20: 561-74. [CrossRef]

Eisend, Martin. 2006. Two-sided advertising: A meta-analysis. International Journal of Research in Marketing 23: 187-98. [CrossRef]

Eisend, Martin. 2007. Understanding two-sided persuasion: An empirical assessment of theoretical approaches. Psychology $\mathcal{E}$ Marketing 24: $615-40$.

Eisend, Martin. 2008. Dual-processing and message sidedness: The effects of involvement in two-sided advertising. Marketing Theory and Applications. AMA Winter Educators' Conference Proceedings 19: 168-69.

Eisend, Martin. 2010. Explaining the joint effect of source credibility and negativity of information in two-sided messages. Psychology E Marketing 27: 1032-49.

Eisend, Martin. 2013. The moderating influence of involvement on two-sided advertising effects. Psychology E Marketing 30: 566-75.

Etgar, Michael, and Stephen A. Goodwin. 1982. One-sided versus two-sided comparative message appeals for new brand introductions. Journal of Consumer Research 8: 460-65. [CrossRef]

Fang, Tony. 2005-2006. From “Onion" to “Ocean." Paradox and Change in National Cultures. International Studies of Management $\mathcal{E}$ Organization 35: 71-90.

Fang, Tony. 2012. Yin Yang: A new perspective on culture. Management and Organization Review 8: 25-50. [CrossRef]

Fang, Tony. 2014. Understanding Chinese culture and communication: The Yin Yang approach. Global Leadership Practices 12: 171-87.

Fang, Tony, and Guy Olivier Faure. 2011. Chinese communication characteristics: A Yin Yang perspective. International Journal of Intercultural Relations 35: 320-33. [CrossRef]

Fornell, Claes, and David F. Larcker. 1981. Evaluating structural equation models with unobservable variables and measurement error. Journal of Marketing Research 18: 39-50. [CrossRef]

Gardner, Meryl Paula. 1985. Does attitude toward the ad affect brand attitude under a brand evaluation set? Journal of Marketing Research 22: 192-98. [CrossRef]

Graham, Angus Charles. 1986. Yin-Yang and the Nature of Correlative Thinking. Singapore: Institute of East Asian Philosophies, vol. 6.

Greer, Jennifer D. 2003. Evaluating the credibility of online information: A test of source and advertising influence. Mass Communication and Society 6: 11-28. [CrossRef]

Hair, Michael L., and Samuel D. Bond. 2018. Attribute dismissal and valence effects in preferential decision processing. Journal of Behavioral Decision Making 31: 164-78. [CrossRef]

Hale, Jerold L., Brian J. Householder, and Kathryn L. Greene. 2002. The theory of reasoned action. In The Persuasion Handbook: Developments in Theory and Practice. Edited by James Price Dillard and Michael Pfau. London: SAGE Publications, vol. 14, pp. 259-86.

Hayes, Andrew F. 2013. Introduction to Mediation, Moderation, and Conditional Process Analysis: A Regression-Based Approach. New York: Guilford Press.

Hayes, Andrew F., and Kristopher J. Preacher. 2014. Statistical mediation analysis with a multicategorical independent variable. British Journal of Mathematical and Statistical Psychology 67: 451-70. [CrossRef]

Hovland, Carl I. 1954. Effects of the mass media of communication. In Handbook of Social Psychology, 5th ed. Edited by Susan T. Fiske, Daniel T. Gilbert and Gardner Lindzey. Hoboken: Wiley, vol. 2, pp. 1062-103.

Huertas, Melby Karina Zuniga, and Eduardo Kalil Hanna. 2020. How Self-confidence Moderates the Effect of Two-sided Messages on Purchase Intention. Journal of Creative Communications 15: 235-54. [CrossRef]

Hunt, James M., and Michael F. Smith. 1987. The persuasive impact of two-sided selling appeals for an unknown brand name. Journal of the Academy of Marketing Science 15: 11-18. [CrossRef]

Jing, Runtian, and Andrew H. Van de Ven. 2014. A Yin-Yang Model of Organizational Change: The Case of Chengdu Bus Group. Management and Organization Review 10: 29-54. [CrossRef] 
Kamins, Michael A., and Henry Assael. 1987. Two-sided versus one-sided appeals: A cognitive perspective on argumentation, source derogation, and the effect of disconfirming trial on belief change. Journal of Marketing Research 24: 29-39. [CrossRef]

Kamins, Michael A., and Lawrence J. Marks. 1987. Advertising puffery: The impact of using two-sided claims on product attitude and purchase intention. Journal of Advertising 16: 6-15. [CrossRef]

Kamins, Michael A., Meribeth J. Brand, Stuart A. Hoeke, and John C. Moe. 1989. Two-sided versus one-sided celebrity endorsements: The impact on advertising effectiveness and credibility. Journal of Advertising 18: 4-10. [CrossRef]

Kao, Danny Tengti. 2011. Message sidedness in advertising: The moderating roles of need for cognition and time pressure in persuasion. Scandinavian Journal of Psychology 52: 329-40. [CrossRef] [PubMed]

Kees, Jeremy, Christopher Berry, Scot Burton, and Kim Sheehan. 2017. An analysis of data quality: Professional panels, student subject pools, and Amazon's Mechanical Turk". Journal of Advertising 46: 141-55. [CrossRef]

Kim, Kenneth. 2020. Stealing thunder in negative political advertising: The persuasive impact of one-sided and two-sided negative messages on partisan individuals. Journal of Creative Communications 15: 7-18. [CrossRef]

Küster, Franziska, and Martin Eisend. 2016. Time heals many wounds-explaining the immediate and delayed effects of message sidedness. International Journal of Advertising 35: 664-81. [CrossRef]

Lang, Bodo, Christina Lee, and Rami Zwick. 1999. Message sidedness at the brand and product form levels: Overcoming the shortcoming of two-sided messages? Advances in Consumer Research 26: 485-90.

Lavagnino, Alessandra C. 2017. From 'Chinese Characteristics' (Zhongguo Tese 中国特色) to 'Chinese Dream' (Zhongguo Meng 中国 梦)-The Chinese Political Discourse Today. In Understanding China Today. Cham: Springer, pp. 275-87.

Lewis, Marianne W. 2000. Exploring paradox: Toward a more comprehensive guide. Academy of Management Review 25: 760-76. [CrossRef]

Li, Peter Ping. 1998. Towards a geocentric framework of organizational form: A holistic, dynamic and paradoxical approach. Organization Studies 19: 829-61. [CrossRef]

Li, Peter Ping. 2008. Toward a geocentric framework of trust: An application to organizational trust. Management and Organization Review 4: 413-39. [CrossRef]

Li, Peter Ping. 2012. Toward an integrative framework of indigenous research: The geocentric implications of Yin-Yang Balance. Asia Pacific Journal of Management 29: 849-72. [CrossRef]

Li, Peter Ping. 2016. Global implications of the indigenous epistemological system from the east. Cross Cultural E Strategic Management 23: 42-77.

Li, Xin, Verner Worm, and Peihong Xie. 2018. Is Yin-Yang superior for paradox re-search? Cross Cultural \& Strategic Management 25: 501-14.

Li, Min-Qian, Yi-Chen Lee, and Ling-Ling Wu. 2020. Sidedness Effect of Online Review. PACIS 2020 Proceedings. 125. Available online: https:/ / aisel.aisnet.org/pacis2020/125 (accessed on 11 June 2021).

MacInnis, Deborah J., C. Whan Park, and Joseph W. Priester. 2014. Handbook of Brand Relationships. London: Routledge.

MacKenzie, Scott B., and Richard J. Lutz. 1989. An empirical examination of the structural antecedents of attitude toward the ad in an advertising pretesting context. Journal of Marketing 53: 48-65. [CrossRef]

Madden, Thomas J., Chris T. Allen, and Jacquelyn L. Twible. 1988. Attitude toward the ad: An assessment of diverse measurement indices under different processing "sets". Journal of Marketing Research 25: 242-52.

Mattsson, Lars-Gunnar, and Annika Tidström. 2015. Applying the principles of Yin-Yang to market dynamics: On the duality of cooperation and competition. Marketing Theory 15: 347-64. [CrossRef]

McSweeney, Brendan. 2015. Hall, Hofstede, Huntington, Trompenaars, GLOBE: Common foundations, common flaws. In Transculturalism and Business in the BRIC States. London: Routledge, pp. 39-84.

Mittal, Banwari. 1990. The relative roles of brand beliefs and attitude toward the ad as mediators of brand attitude: A second look. Journal of Marketing Research 27: 209-19. [CrossRef]

Monga, Alokparna Basu, and Deborah Roedder John. 2004. Consumer response to brand extensions: Does culture matter? ACR North American Advances 31: 216-22.

Moon, Hakil, and David E. Sprott. 2016. Ingredient branding for a luxury brand: The role of brand and product fit. Journal of Business Research 69: 5768-74. [CrossRef]

Nisbett, Richard E. 2003. The Geography of Thought: How Asians and Westerners Think Differently ... and Why. London: Nicholas Brealey Publishing.

Nisbett, Richard E., Kaiping Peng, Incheol Choi, and Ara Norenzayan. 2001. Culture and systems of thought: Holistic versus analytic cognition. Psychological Review 108: 291-310. [CrossRef]

Nunnally, Jum C., and Ira H. Bernstein. 1994. Psychometric Theory. New York: McGraw-Hill.

O'Keefe, Daniel J. 1999. How to handle opposing arguments in persuasive messages: A meta-analytic review of the effects of one-sided and two-sided messages. Annals of the International Communication Association 22: 209-49. [CrossRef]

Park, Jong-Won, and Manoj Hastak. 1994. Memory-based product judgments: Effects of involvement at encoding and retrieval. Journal of Consumer Research 21: 534-47. [CrossRef]

Pauluzzo, Rubens. 2020. Applying Yin Yang Wisdom in Western Organizational Settings: Using Interviews, Documents, and Field Observations for Cross-Cultural Research. London: SAGE Publications Ltd. 
Pauluzzo, Rubens, Marta Guarda, Laura De Pretto, and Tony Fang. 2018. Managing paradoxes, dilemmas, and change: A case study to apply the Yin Yang wisdom in Western organizational settings. Cross Cultural E Strategic Management 25: 257-75.

Peng, Kaiping. 1997. Naive Dialecticism and Its Effects on Reasoning and Judgment about Contradiction. Doctoral dissertation, University of Michigan, Ann Arbor, MI, USA.

Peng, Kaiping, and Richard E. Nisbett. 1999. Culture, dialectics, and reasoning about contradiction. American Psychologist 54: 741-54. [CrossRef]

Pizzutti, Cristiane, Kenny Basso, and Manuela Albornoz. 2016. The effect of the discounted attribute importance in two-sided messages. European Journal of Marketing 50: 1703-25. [CrossRef]

Podsakoff, Philip M., Scott B. MacKenzie, Jeong-Yeon Lee, and Nathan P. Podsakoff. 2003. Common method biases in behavioral research: A critical review of the literature and recommended remedies. Journal of Applied Psychology 88: 879-903. [CrossRef] [PubMed]

Putrevu, Sanjay, and Kenneth R. Lord. 1994. Comparative and noncomparative advertising: Attitudinal effects under cognitive and affective involvement conditions. Journal of Advertising 23: 77-91. [CrossRef]

Qi, Dai, Liang Liang, Cao Zhongpeng, and Wu Jianlin. 2010a. Responses to two-sided advertising: The moderating effects of need for cognition. Paper presented at International Conference of Engineering Business Management (EBM 2010), Chengdu, China, March 24-26; pp. 2456-59.

Qi, Dai, Liang Liang, Wu Jianlin, and Cao Zhongpeng. 2010b. The Impact of Message Sidedness on Online Ads Effectiveness: The Moderating Role of Involvement. Paper presented at 2010 International Conference on E-Business and E-Government IEEE, Guangzhou, China, May 7-9; pp. 3164-67.

Shimp, Terence A. 1981. Attitude toward the ad as a mediator of consumer brand choice. Journal of Advertising 10: 9-48. [CrossRef]

Smith, Robert E., and Shelby D. Hunt. 1978. Attributional processes and effects in promotional situations. Journal of Consumer Research 5: 149-58. [CrossRef]

Spears, Nancy, and Surendra N. Singh. 2004. Measuring attitude toward the brand and purchase intentions. Journal of Current Issues $\mathcal{E}$ Research in Advertising 26: 53-66.

Sugitani, Yoko. 2014. A Brand Attitude Comparison between East Asians and Westerners: Exploring the Role of Emotion and Cognition on Attitude Formation. Paper presented at the 2014 Global Marketing Conference, Singapore, July 15-18; pp. 1484-89.

Swinyard, William R. 1981. The interaction between comparative advertising and copy claim variation. Journal of Marketing Research 18: 175-86. [CrossRef]

Tian, Chenshan. 2002. Tongbian in the Chinese reading of dialectical materialism. Philosophy East and West 52: 126-44. [CrossRef]

Trifts, Valerie, and Gerald Häubl. 2003. Information availability and consumer preference: Can online retailers benefit from providing access to competitor price information? Journal of Consumer Psychology 13: 149-59. [CrossRef]

Uribe, Rodrigo, Cristian Buzeta, and Milenka Velásquez. 2016. Sidedness, commercial intent and expertise in blog advertising. Journal of Business Research 69: 4403-10. [CrossRef]

Zhou, Nan, and Russell W. Belk. 2004. Chinese consumer readings of global and local advertising appeals. Journal of Advertising 33: 63-76. [CrossRef]

Zolfani, Sarfaraz Hashemkhani, Edmundas Kazimieras Zavadskas, and Zenonas Turskis. 2013. Design of products with both International and Local perspectives based on Yin-Yang balance theory and SWARA method. Economic Re-search-Ekonomska istraživanja 26: 153-66. [CrossRef] 